\title{
Addition/Oxidative Rearrangement of 3- Furfurals and 3-Furyl Imines: New Approaches to Substituted Furans and Pyrroles
}

\author{
Ann Rowley Kelly, Michael H. Kerrigan and PatrickJ. Walsh*
}

Contribution from the P. Roy and Diana T. Vagelos Laboratories, Department of

Chemistry, University of Pennsylvania, 231 South 34th Street, Philadelphia, PA 19104-

6323

pwalsh@sas.upenn.edu

\section{Supporting Information}


General Methods. All carbonyl addition reactions involving diorganozinc, Grignard, or organolithium reagents were carried out under a nitrogen atmosphere using standard Schlenk or vacuum line techniques with oven-dried glassware. The progress of all reactions was monitored by thin-layer chromatography. Diethylzinc was a gift of Akzo Chemical. All other zinc reagents and $N$-furan-3-ylmethylene-4-methylbenzenesulfonamide (11) were prepared by literature methods. ${ }^{1-4}$ THF and $\mathrm{Et}_{2} \mathrm{O}$ were dried over sodium and benzophenone and distilled. All aldehydes were distilled prior to use and stored under $\mathrm{N}_{2}$. NBS was purchased from Acros and used as recieved. Unless otherwise specified, all chemicals were obtained from Acros, Aldrich, or GFS Chemicals, and all solvents were purchased from Fischer Scientific. The ${ }^{1} \mathrm{H}$ NMR and ${ }^{13} \mathrm{C}\left\{{ }^{1} \mathrm{H}\right\}$ NMR spectra were obtained on Bruker AM-500, DMX-360 and DMX 300 Fourier transform NMR spectrometers at 500,360, and $300 \mathrm{MHz}$ for ${ }^{1} \mathrm{H}$ and 125, 90, $75 \mathrm{MHz}$ for ${ }^{13} \mathrm{C}\left\{{ }^{1} \mathrm{H}\right\}$ NMR, respectively. Chemical shifts are reported in units of parts per million downfield from tetramethylsilane, and all coupling constants are reported in Hertz. A vertical asymptote at $4.5 \mathrm{ppm}$ appears on some ${ }^{1} \mathrm{H}$ NMR spectra, which seemed to be an issue with a previous version of iNMR software. The infrared spectra were obtained using a Perkin-Elmer Spectrum 100 series FT-IR spectrometer. Thin-layer chromatography was performed on Whatman precoated silica gel $60 \AA$ plates $(250 \mu \mathrm{m}$ layer thickness) with fluorescent indicator and visualized by ultra-violet light or by staining with ceric ammonium molybdate stain. Silica gel (230-400 mesh, Silicycle) was used for air-flashed chromatography. Deactivated silica gel was prepared by premixing silica gel $(800 \mathrm{~mL})$ with triethylamine $(20 \mathrm{~mL})$. Analysis of enantiomeric excess was performed using an Agilent 1100 Series HPLC and Chiralpak AD or AD-H columns or 
by chiral capillary gas chromatography on a Hewlett-Packard 6890 GC with a Beta $\operatorname{DEX}^{\mathrm{TM}} 120$ fused silica column ( $30 \mathrm{~m}$ x $0.25 \mathrm{~mm}$ x $0.25 \mu \mathrm{m}$ film thickness).

\section{$\underline{\text { Furyl Alcohols }}$}

\section{General Procedure A: Synthesis of Furyl Alcohols from 3-Furfural.}

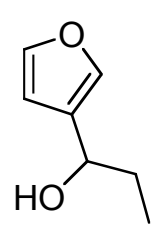

1-Furan-3-yl-propan-1-ol (2a). General Procedure A. Under a nitrogen atmosphere, a $25 \mathrm{~mL}$ purged flask was charged with 3-furfural $(84 \mu \mathrm{L}, 1.0$ mmol) and THF $(1.0 \mathrm{~mL})$ and cooled to $0{ }^{\circ} \mathrm{C}$. A solution of ethyl magnesium bromide ( $0.5 \mathrm{~mL}, 3.0 \mathrm{M}$ solution in ether) was added dropwise. The reaction was stirred at $0{ }^{\circ} \mathrm{C}$ for $3 \mathrm{~h}$ and quenched with saturated aqueous $\mathrm{NH}_{4} \mathrm{Cl}$. After the organic and aqueous layers were separated, the aqueous layer was extracted with $\mathrm{Et}_{2} \mathrm{O}(3 \mathrm{X} 10 \mathrm{~mL})$. The combined organic layers were dried over $\mathrm{MgSO}_{4}$ and filtered. The filtrate was concentrated in vacuo, and the residue was chromatographed on silica (10\% EtOAc in hexanes) to afford the title compound as a colorless oil in 98\% yield (123 mg, 0.98 mmol). The spectra for this compound were identical to previously reported literature data. ${ }^{5}$

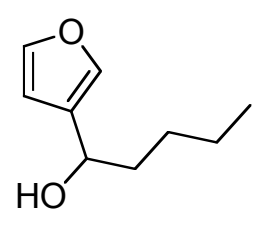

1-Furan-3-yl-pentan-1-ol (2b). This compound was prepared by General Procedure A using $n$-butyl lithium $(0.6 \mathrm{~mL}, 2.5 \mathrm{M}$ solution in hexane), 3-furfural ( $84 \mu \mathrm{L}, 1.0 \mathrm{mmol})$, and THF (1.0 mL) were added to the reaction vessel. After the usual workup, the crude product was purified by column chromatography on deactivated silica (10\% EtOAc in hexanes) to afford the title 
compound as a colorless oil in $95 \%$ yield $(146 \mathrm{mg}, 0.95 \mathrm{mmol})$. The spectra for this compound match with previously reported literature data. ${ }^{6}$

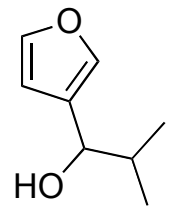

1-Furan-3-yl-2-methyl-propan-1-ol (2c). This compound was prepared by General Procedure A using isopropyl magnesium bromide $(1.5 \mathrm{~mL}, 1.0 \mathrm{M}$ solution in THF), 3-furfural $(84 \mu \mathrm{L}, 1.0 \mathrm{mmol})$, and THF $(1.0 \mathrm{~mL})$ were added to the reaction vessel. After the usual workup, the crude product was purified by column chromatography on deactivated silica (10\% EtOAc in hexanes) to afford the title compound as a colorless oil in $91 \%$ yield $(127 \mathrm{mg}, 0.91 \mathrm{mmol}) .{ }^{1} \mathrm{H}$ NMR $\left(\mathrm{CDCl}_{3}, 360\right.$ MHz) $\delta 7.34(\mathrm{~d}, J=0.6 \mathrm{~Hz}, 1 \mathrm{H}), 7.31(\mathrm{~d}, J=0.6 \mathrm{~Hz}, 1 \mathrm{H}), 6.33(\mathrm{~d}, J=0.6 \mathrm{~Hz}, 1 \mathrm{H}), 4.33$ (d, $J=6.9 \mathrm{~Hz}, 1 \mathrm{H}), 1.98($ br s, $1 \mathrm{H}), 1.87(\mathrm{~m}, 1 \mathrm{H}), 0.95(\mathrm{~d}, J=5.4 \mathrm{~Hz}, 3 \mathrm{H})$, and $0.83(\mathrm{~d}, J$ $=5.4 \mathrm{~Hz}, 3 \mathrm{H}) \mathrm{ppm} ;{ }^{13} \mathrm{C}\left\{{ }^{1} \mathrm{H}\right\} \mathrm{NMR}\left(\mathrm{CDCl}_{3}, 90 \mathrm{MHz}\right): \delta$ 143.0, 139.5, 127.8, 108.8, 72.4, $34.2,18.6$, and $18.0 \mathrm{ppm}$; IR (neat) 3402, 2961, 2873, 2358, 1651, 1557, 1557, 1503, $1469,1383,1258,1160,1123,1067,1023,974,918,874$, and $791 \mathrm{~cm}^{-1}$. HRMS-CI $\mathrm{m} / \mathrm{z}$ $140.0829\left[\mathrm{M}^{+}\right.$; calcd for $\left.\mathrm{C}_{8} \mathrm{H}_{12} \mathrm{O}_{2}: 140.0837\right]$.

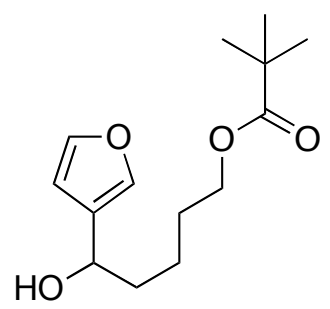

2,2-Dimethyl propionic acid ester- furan-3-yl-butanol (2d). This compound was prepared by General Procedure A using $\left(\mathrm{PivO}\left(\mathrm{CH}_{2}\right)_{4}\right)_{2} \mathrm{Zn}^{2}(2 \mathrm{~mL}, 1.0 \mathrm{M}$ solution in toluene), 3-furfural (84 $\mu \mathrm{L}, 1.0 \mathrm{mmol})$, and THF $(1.0 \mathrm{~mL})$ were added to the reaction vessel. After the usual workup, the crude product was purified by column chromatography on deactivated silica (10\% EtOAc in hexanes) to afford the title compound as a colorless oil in $83 \%$ yield (279 mg, $0.83 \mathrm{mmol}) .{ }^{1} \mathrm{H}$ NMR $\left(\mathrm{CDCl}_{3}, 500 \mathrm{MHz}\right) \delta 7.38(\mathrm{~s}, 2 \mathrm{H}), 6.40$ (s, 
$1 \mathrm{H}), 4.65(\mathrm{t}, J=6.5 \mathrm{~Hz}, 1 \mathrm{H}), 4.05(\mathrm{t}, J=7.0 \mathrm{~Hz}, 2 \mathrm{H}), 2.04(\mathrm{br} \mathrm{s}, 1 \mathrm{H}) 1.74(\mathrm{~m}, 2 \mathrm{H})$, $1.40(\mathrm{~m}, 1 \mathrm{H}), 1.38(\mathrm{~m}, 1 \mathrm{H})$, and $1.17(\mathrm{~s}, 9 \mathrm{H}) \mathrm{ppm} ;{ }^{13} \mathrm{C}\left\{{ }^{1} \mathrm{H}\right\} \mathrm{NMR}\left(\mathrm{CDCl}_{3}, 125 \mathrm{MHz}\right): \delta$ $178.7,143.3,139.0,129.0,108.4,66.6,64.1,37.3,37.2,28.4,27.1$, and $22.9 \mathrm{~cm}^{-1}$. IR (neat) $3440,2958,2870,2350,2077,1724,1650,1555,1503,1480,1461,1398,1365$, 1286, 1159, 1063, 1022, and $874 \mathrm{~cm}^{-1}$. HRMS-CI $\mathrm{m} / z$ 254.1505 [M-OH ${ }^{+}$; calcd for $\left.\mathrm{C}_{14} \mathrm{H}_{20} \mathrm{O}_{3}{ }^{-}: 254.1518\right]$.

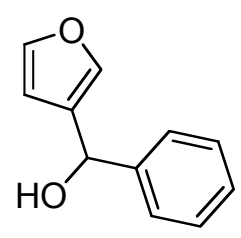

Furan-3-yl-phenyl-methanol (2e). This compound was prepared by General Procedure A using phenyllithium $(0.75 \mathrm{~mL}, 2.0 \mathrm{M}$ solution in THF), 3-furfural (84 $\mu \mathrm{L} \mathrm{L}, 1.0 \mathrm{mmol})$, and THF (1.0 mL) were added to the reaction vessel. After the usual workup, the crude product was purified by column chromatography on deactivated silica (10\% EtOAc in hexanes) to afford the title compound as a colorless oil in $94 \%$ yield $(163.5 \mathrm{mg}, 0.94 \mathrm{mmol})$. The spectra for this compound match with previously reported literature data. ${ }^{7}$

Synthesis of Furan-3-yl-phenyl-methanol (2e) from 3-Bromofuran. Under a nitrogen atmosphere, $n$-BuLi $(0.4 \mathrm{~mL}, 2.5 \mathrm{M}$ in hexanes, $1.0 \mathrm{mmol})$ was added dropwise to a stirred solution of 3-bromofuran $(108 \mu \mathrm{L}, 1.2 \mathrm{mmol})$ in dry THF $(1 \mathrm{~mL})$ at $-78{ }^{\circ} \mathrm{C}$. After $15 \mathrm{~m}$, benzaldehyde $(101 \mu \mathrm{L}, 1.0 \mathrm{mmol})$ was added to the solution. The reaction mixture was stirred at $-78{ }^{\circ} \mathrm{C}$ until complete by TLC and quenched with saturated aqueous $\mathrm{NH}_{4} \mathrm{Cl}$. The organic and aqueous layers were separated, and the aqueous layer was extracted with $\mathrm{Et}_{2} \mathrm{O}(3 \mathrm{X} 10 \mathrm{~mL})$. The combined organic layers were dried over $\mathrm{MgSO}_{4}$ and filtered. The filtrate was concentrated in vacuo and the residue was purified by 
column chromatography on deactivated silica (10\% EtOAc in hexanes) to afford the title compound as a colorless oil in $90 \%$ yield $(157 \mathrm{mg}, 0.90 \mathrm{mmol})$. The spectra for this compound were identical with previously reported literature data. ${ }^{7}$

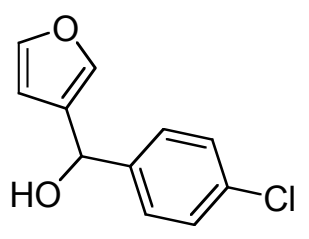

2-(4-Chloro-phenyl)-furan-3-yl-methanol (2f). This compound was prepared by General Procedure A after an in situ generation of the Grignard reagent. Under a nitrogen atmosphere, a dry $25 \mathrm{~mL}$ flask with stirbar was charged with 1-bromo, 4-chlorophenyl (383 mg, $2.0 \mathrm{mmol})$, magnesium turnings (48.6 mg, $2.0 \mathrm{mmol}), 1$ crystal of $\mathrm{I}_{2}$, and THF $(4 \mathrm{~mL})$. The flask was heated to $50{ }^{\circ} \mathrm{C}$ while stirring. When the magnesium had dissolved the reaction was allowed to cool to $0{ }^{\circ} \mathrm{C}$, after which 3-furfural $(84 \mu \mathrm{L} \mathrm{L}, 1.0 \mathrm{mmol})$ was added to the reaction vessel. The reaction was stirred at $0{ }^{\circ} \mathrm{C}$ for $3 \mathrm{~h}$ and quenched with saturated aqueous $\mathrm{NH}_{4} \mathrm{Cl}$. After the organic and aqueous layers were separated, the aqueous layer was extracted with $\mathrm{Et}_{2} \mathrm{O}(3 \times 10 \mathrm{~mL})$. The combined organic layers were dried over $\mathrm{MgSO}_{4}$ and filtered. The filtrate was concentrated in vacuo and the residue was chromatographed on silica (10\% EtOAc in hexanes) to afford the title compound as a colorless oil in $99 \%$ yield (206.5 mg, $0.99 \mathrm{mmol}) ;{ }^{1} \mathrm{H} \mathrm{NMR}\left(\mathrm{CDCl}_{3}, 300 \mathrm{MHz}\right) \delta 7.36(\mathrm{~s}, 1 \mathrm{H}), 7.31(\mathrm{~s}, 4 \mathrm{H})$, $7.22(\mathrm{~s}, 1 \mathrm{H}), 6.28(\mathrm{~s}, 1 \mathrm{H}), 5.68(\mathrm{~s}, 1 \mathrm{H})$, and $2.71(\mathrm{br} \mathrm{s}, 1 \mathrm{H}) \mathrm{ppm} ;{ }^{13} \mathrm{C}\left\{{ }^{1} \mathrm{H}\right\} \mathrm{NMR}\left(\mathrm{CDCl}_{3}\right.$, $75 \mathrm{MHz}$ ): $\delta$ 144.9, 142.8, 140.8, 134.7, 130.0, 129.8, 110.1, and $69.5 \mathrm{ppm}$; IR (neat) $3337,2970,2941,2676,2346,2336,2089,1904,1782,1673,1588,1504,1489,1425$, $1355,1266,1217,1162,1088$, and 1028. $\mathrm{cm}^{-1}$. HRMS-CI $\mathrm{m} / z 191.0268\left[{\mathrm{M}-\mathrm{OH}^{+} \text {; calcd }}^{2}\right.$ for $\mathrm{C}_{11} \mathrm{H}_{8} \mathrm{OCl}$ : 191.0258]. 


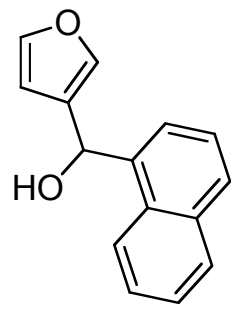

2-(1-napthal)-furan-3-yl-methanol (2g). This compound was prepared by General Procedure A after an in situ generation of the Grignard reagen using 1-bromonapthalene ( $278 \mu \mathrm{L}, 2.0 \mathrm{mmol}))$, magnesium turnings (48.6 mg, $2.0 \mathrm{mmol}), 3$-furfural $(84 \mu \mathrm{L}, 1.0 \mathrm{mmol})$, and THF $(4 \mathrm{~mL})$ were added to the reaction vessel. After the usual workup, the crude product was purified by column chromatography on deactivated silica (10\% EtOAc in hexanes) to afford the title compound as a colorless oil in $95 \%$ yield $(213 \mathrm{mg}, 0.95 \mathrm{mmol}) .{ }^{1} \mathrm{H} \mathrm{NMR}\left(\mathrm{CDCl}_{3}\right.$, $500 \mathrm{MHz}) \delta 8.07(\mathrm{~d}, J=8.0 \mathrm{~Hz}, 1 \mathrm{H}), 7.94(\mathrm{~d}, J=7.5 \mathrm{~Hz}, 1 \mathrm{H}), 7.87(\mathrm{~d}, J=8.0 \mathrm{~Hz}, 1 \mathrm{H})$, $7.69(\mathrm{~d}, J=7.0 \mathrm{~Hz}, 1 \mathrm{H}), 7.53(\mathrm{~m}, 4 \mathrm{H}), 7.39(\mathrm{~s}, 1 \mathrm{H}), 7.28(\mathrm{~s}, 1 \mathrm{H}), 6.41(\mathrm{~s}, 1 \mathrm{H}), 6.38(\mathrm{~s}$, 1H), 3.23 (br s, 1H) ppm; ${ }^{13} \mathrm{C}\left\{{ }^{1} \mathrm{H}\right\} \mathrm{NMR}\left(\mathrm{CDCl}_{3}, 90 \mathrm{MHz}\right): \delta 143.2,140.3,138.4,133.9$, $130.5,128.8,128.5,126.1,125.7,125.4,123.8,123.7,109.6$, and $66.8 \mathrm{ppm}$; IR (neat) $3345,3345,3146,3049,3011,2968,2924,2360,2345,1953,1824,1773,1718,1702$, $1624,1597,1509,1458,1388,1316,1260,1227,1156,1054,1019,996$, and $960 \mathrm{~cm}^{-1}$. HRMS-CI $m / z 224.0839\left[\mathrm{M}^{+}\right.$; calcd for $\mathrm{C}_{15} \mathrm{H}_{12} \mathrm{O}_{2}: 224.0837$ ].

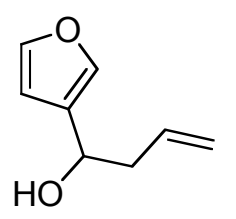

2-Allyl-furan-3-yl-methanol (2h). This compound was prepared by General Procedure A using allyl magnesium bromide $(1.5 \mathrm{~mL}, 1.0 \mathrm{M}$ solution in THF), 3-furfural $(84 \mu \mathrm{L}, 1.0 \mathrm{mmol})$, and THF $(1 \mathrm{~mL})$ were added to the reaction vessel. After the usual workup, the crude product was purified by column chromatography on deactivated silica (10\% EtOAc in hexanes) to afford the title compound as a colorless oil in $89 \%$ yield $(122.8 \mathrm{mg}, 0.89 \mathrm{mmol}) .{ }^{1} \mathrm{H} \mathrm{NMR}\left(\mathrm{CDCl}_{3}, 360\right.$ MHz) $\delta 7.44(\mathrm{t}, J=1.6 \mathrm{~Hz}, 2 \mathrm{H}), 6.34(\mathrm{~d}, J=1.6 \mathrm{~Hz}, 1 \mathrm{H}), 5.75(\mathrm{~m}, 1 \mathrm{H}), 5.08(\mathrm{~m}, 2 \mathrm{H})$, $4.62(\mathrm{t}, J=3.6 \mathrm{~Hz}, 1 \mathrm{H}), 2.69(\mathrm{br} \mathrm{s}, 1 \mathrm{H})$ and $2.43(\mathrm{t}, J=7.2 \mathrm{~Hz}, 2 \mathrm{H}) \mathrm{ppm} ;{ }^{13} \mathrm{Ci} \mathrm{NMR}$ 
$\left(\mathrm{CDCl}_{3}, 90 \mathrm{MHz}\right): \delta 143.2,139.0,134.2,128.5,118.2,108.6,66.0$, and $42.3 \mathrm{ppm}$; IR (neat) 3358, 3149, 3076, 3003, 2978, 2933, 2660, 2547, 2356, 2316, 2225, 2030, $1841,1698,1641,1595,1502,1433,1416,1392,1336,1298,1246,1227,1159,1108$, and $1025 \mathrm{~cm}^{-1}$. HRMS-CI $\mathrm{m} / z$ 138.0685[M+calcd for $\left.\mathrm{C}_{8} \mathrm{H}_{10} \mathrm{O}_{2}: 138.0680\right]$.

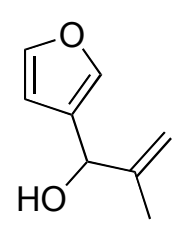

1-Furan-3-yl-2-methyl-prop-2-en-1-ol (2i). This compound was prepared by General Procedure A after the in situ generation of the organolithium reagent. Under a nitrogen atmosphere, 1-bromo-2-methyl-2-propene $(130 \mu \mathrm{L}$, $1.5 \mathrm{mmol})$ and THF $(4 \mathrm{~mL})$ were added to the flask and cooled to $-78{ }^{\circ} \mathrm{C} . n$-Butyl lithium (0.6 mL, $1.5 \mathrm{mmol}, 2.5 \mathrm{M}$ solution in hexanes) was added dropwise while stirring. After $1 \mathrm{~h}, 3$-furfural $(84 \mu \mathrm{L}, 1.0 \mathrm{mmol})$ was added to the reaction vessel. The reaction was stirred at $-78{ }^{\circ} \mathrm{C}$ for $3 \mathrm{~h}$ and quenched with saturated aqueous $\mathrm{NH}_{4} \mathrm{Cl}$. After the organic and aqueous layers were separated, the aqueous layer was extracted with $\mathrm{Et}_{2} \mathrm{O}(3 \mathrm{X}$ 10mL). The combined organic layers were dried over $\mathrm{MgSO}_{4}$ and filtered. The filtrate was concentrated in vacuo and the residue was chromatographed on silica (10\% EtOAc in hexanes) to afford the title compound as a colorless oil in 90\% yield (124 mg, 0.90 mmol); ${ }^{1} \mathrm{H} \mathrm{NMR}\left(\mathrm{CDCl}_{3}, 300 \mathrm{MHz}\right) \delta 7.39(\mathrm{~m}, 2 \mathrm{H}), 6.35$ (s, 1H), $5.16(\mathrm{~s}, 1 \mathrm{H}), 5.11$ (s, 1H), $4.96(\mathrm{~s}, 1 \mathrm{H})$, and $1.71(\mathrm{~s}, 3 \mathrm{H}) \mathrm{ppm} ;{ }^{13} \mathrm{C}\left\{{ }^{1} \mathrm{H}\right\} \mathrm{NMR}\left(\mathrm{CDCl}_{3}, 125 \mathrm{MHz}\right): \delta 146.3$, 143.2, 139.8, 127.0, 111.2, 109.0, 70.9, and 18.1 ppm; IR (neat) 3375, 2972, 2918, 2861, 2657, 2360, 2310, 2225, 2033, 1812, 1700, 1653, 1593, 1502, 1448, 1377, 1236, 1213, $1158,1020,994,945,904,874,832,773,730,690,658,627$, and $600 \mathrm{~cm}^{-1}$. LRMS-CI $m / z 123.047$ [M-Me ${ }^{+}$; calcd for $\left.\mathrm{C}_{7} \mathrm{H}_{7} \mathrm{O}_{2}: 123.045\right]$. 


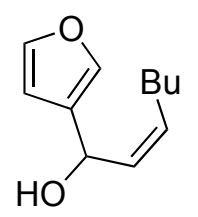

1-Furan-3-yl- $Z$-hex-2-en-1-ol (2j). Under a nitrogen atmosphere, a dry 10 $\mathrm{mL}$ schlenk flask with stirbar was loaded with $\mathrm{Cy}_{2} \mathrm{BH}(267 \mathrm{mg}, 1.5 \mathrm{mmol}){ }^{3}$ At $0{ }^{\circ} \mathrm{C}$, THF $(1 \mathrm{~mL})$ and 1-bromohexyne $(191 \mu \mathrm{L}, 1.5 \mathrm{mmol})$ were added, allowed to warm to room temp and stirred for $30 \mathrm{~m}$. At $-78{ }^{\circ} \mathrm{C}, t$-butyl lithium $(0.88 \mathrm{~mL}$, $1.7 \mathrm{M}$ in hexanes) was added dropwise. After stirring for $30 \mathrm{~m}$, the temperature was raised to $0{ }^{\circ} \mathrm{C}$ and stirred for an additional $30 \mathrm{~m}$. The solvent was removed in vacuo. Cooling to $-78{ }^{\circ} \mathrm{C}$, hexanes $(1 \mathrm{~mL})$ and $\mathrm{Et}_{2} \mathrm{Zn}(1.5 \mathrm{~mL}, 1.5 \mathrm{mmol}, 1.0 \mathrm{M}$ solution in hexanes) were added to the flask. After $30 \mathrm{~m}, 3$-furfural $(84 \mu \mathrm{L}, 1.0 \mathrm{mmol})$ was added to the reaction vessel. The reaction was stirred at $0{ }^{\circ} \mathrm{C}$ for $8 \mathrm{~h}$ and quenched with saturated aqueous $\mathrm{NH}_{4} \mathrm{Cl}$. After the organic and aqueous layers were separated, the aqueous layer was extracted with $\mathrm{Et}_{2} \mathrm{O}(3 \mathrm{X} 10 \mathrm{~mL})$. The combined organic layers were dried over $\mathrm{MgSO}_{4}$ and filtered. The filtrate was concentrated in vacuo and the residue was chromatographed on silica (5-10\% EtOAc in hexanes) to afford the title compound as a colorless oil in $92 \%$ yield (166 mg, $0.92 \mathrm{mmol}) .{ }^{1} \mathrm{H} \mathrm{NMR}\left(\mathrm{CDCl}_{3}, 500 \mathrm{MHz}\right) \delta 7.41(\mathrm{~d}, J=10 \mathrm{~Hz}, 2 \mathrm{H}), 6.44$ (s, 1H), $5.64(\mathrm{~m}, 2 \mathrm{H}), 5.53(\mathrm{~d}, J=3.7 \mathrm{~Hz}, 1 \mathrm{H}), 2.18(\mathrm{~m}, 2 \mathrm{H}), 1.78(\mathrm{~d}, J=4.5 \mathrm{~Hz}, 1 \mathrm{H})$, $1.38(\mathrm{~m}, 4 \mathrm{H})$, and $0.93(\mathrm{t}, J=7.0 \mathrm{~Hz}, 1 \mathrm{H}) \mathrm{ppm} ;{ }^{13} \mathrm{C}\left\{{ }^{1} \mathrm{H}\right\} \mathrm{NMR}\left(\mathrm{CDCl}_{3}, 75 \mathrm{MHz}\right): \delta$ $143.4,139.0,132.7,130.8,128.4,108.8,62.9,31.7,27.3,22.3$, and 13.9 ppm; IR (neat) $3354,3013,2957,2928,2859,1654,1592,1501,1465,1378,1273,1159,1023,935$, 874, 820, 778, and $727 \mathrm{~cm}^{-1}$. HRMS-CI $m / z 162.1038\left[\mathrm{M}-\mathrm{H}_{2} \mathrm{O}^{+}\right.$; calcd for $\mathrm{C}_{11} \mathrm{H}_{14} \mathrm{O}$ : 162.1045].

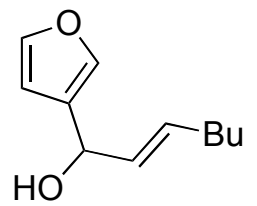

1-Furan-3-yl-E-hex-2-en-1-ol (2k). Under a nitrogen atmosphere, a dry $10 \mathrm{~mL}$ schlenk flask with stirbar was charged with $\mathrm{Cy}_{2} \mathrm{BH}(267 \mathrm{mg}, 1.5$ 
mmol). ${ }^{1} \quad$ At room temperature, hexane $(1 \mathrm{~mL})$ and 1-hexyne $(175 \mu \mathrm{L}, 1.5 \mathrm{mmol})$ were added and stirred for $30 \mathrm{~m}$. In a separate schlenk flask, $\mathrm{LiBr}(1.0 \mathrm{mmol}, 86 \mathrm{mg})$ was added and the flask was heated to $100{ }^{\circ} \mathrm{C}$ under vacuum $(\sim 1-2$ torr $)$ for $1 \mathrm{~h}$. The hydroboration reaction mixture was allowed to cool to room temp and hexanes $(1 \mathrm{~mL})$ and $\mathrm{Et}_{2} \mathrm{Zn}(1.5 \mathrm{~mL}, 1.5 \mathrm{mmol}, 1.0 \mathrm{M}$ solution in hexanes $)$ were added. Cooling to $-78{ }^{\circ} \mathrm{C}$, the vinylzinc solution was cannulated into the flask containing LiBr. After $30 \mathrm{~m}$, the 3furfural $(84 \mu \mathrm{L}, 1.0 \mathrm{mmol})$ was added, and the reaction was warmed to $0{ }^{\circ} \mathrm{C}$. Upon completion, the reaction mixture was quenched with $\mathrm{NaHCO}_{3}$. After the organic and aqueous layers were separated, the aqueous layer was extracted with $\mathrm{Et}_{2} \mathrm{O}(3 \times 10 \mathrm{~mL})$. The combined organic layers were dried over $\mathrm{MgSO}_{4}$ and filtered. The filtrate was concentrated in vacuo and the residue was chromatographed on silica (10\% EtOAc in hexanes) to afford the title compound as a colorless oil in 93\% yield (167 mg, 0.93 mmol); ${ }^{1} \mathrm{H} \mathrm{NMR}\left(\mathrm{CDCl}_{3}, 500 \mathrm{MHz}\right) \delta 7.41(\mathrm{t}, J=1.5 \mathrm{~Hz}, 1 \mathrm{H}), 7.39(\mathrm{~s}, 1 \mathrm{H}), 6.41(\mathrm{~s}, 1 \mathrm{H})$, $5.79(\mathrm{dt}, J=15.0,6.5 \mathrm{~Hz}, 1 \mathrm{H}), 5.71(\mathrm{dd}, J=6.5,15.0 \mathrm{~Hz}, 1 \mathrm{H}), 5.15(\mathrm{~d}, J=6.5 \mathrm{~Hz}, 1 \mathrm{H})$, $2.09(\mathrm{q}, J=6.5 \mathrm{~Hz}, 2 \mathrm{H}), 1.82($ br s, $1 \mathrm{H}), 1.38(\mathrm{~m}, 4 \mathrm{H})$, and $0.91(\mathrm{t}, J=7.0 \mathrm{~Hz}, 3 \mathrm{H}) \mathrm{ppm}$; ${ }^{13} \mathrm{C}\left\{{ }^{1} \mathrm{H}\right\} \mathrm{NMR}\left(\mathrm{CDCl}_{3}, 125 \mathrm{MHz}\right): \delta 143.3,139.1,133.1,131.2,128.3,109.0,68.1,31.8$, 31.2, 22.2, and 13.9 ppm; IR (neat) 3357, 2956, 2926, 2856, 2362, 1716, 1669, 1593, $1501,1465,1377,1159,1089,1064,1023,968,874,792,728$, and $600 \mathrm{~cm}^{-1}$. HRMS-CI $m / z 180.1142\left[\mathrm{M}^{+}\right.$; calcd for $\left.\mathrm{C}_{11} \mathrm{H}_{16} \mathrm{O}_{2}: 180.1150\right]$.

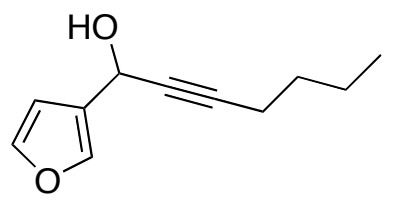

1-Furan-3-yl-hept-2-yn-1-ol (21). This compound was prepared by General Procedure A after the in situ generation of the organolithium reagent. To a solution of 1-hexyne $(289 \mu \mathrm{L}, 2.5 \mathrm{mmol})$ in THF $(4 \mathrm{~mL})$ 
was added $\mathrm{n}$-BuLi (1.0 mL, $2.5 \mathrm{M}$ in hexanes) dropwise at $-78{ }^{\circ} \mathrm{C}$ and stirred for 30 m. Warming to $0{ }^{\circ} \mathrm{C}, 3$-furfural $(167 \mu \mathrm{L}, 2.0 \mathrm{mmol})$ was added dropwise and was allowed to warm to rt over $2 \mathrm{~h}$. The reaction was quenched with $\mathrm{H}_{2} \mathrm{O}$ and $\mathrm{HCl}(2 \mathrm{~N})$. After EtOAc $(2 \mathrm{~mL})$ was added and the organic layer was separated, the aqueous layer was extracted with EtOAc ( 3 X $5 \mathrm{~mL})$. The combined organic layers were washed with brine, dried over $\mathrm{MgSO}_{4}$, and filtered. The filtrate was concentrated in vacuo. The residue was purified by column chromatography on silica (5-20\% EtOAc in hexanes) to afford the title compound as a colorless oil in $92 \%$ yield $(328.5 \mathrm{mg}, 1.84 \mathrm{mmol}) .{ }^{1} \mathrm{H}$ NMR $\left(\mathrm{CDCl}_{3}, 500 \mathrm{MHz}\right) \delta 7.53(\mathrm{~s}, 1 \mathrm{H}), 7.41(\mathrm{~s}, 1 \mathrm{H}), 6.52(\mathrm{~s}, 1 \mathrm{H}), 5.40(\mathrm{~s}, 1 \mathrm{H}), 2.28(\mathrm{dt}$, $J=1.8 \mathrm{~Hz}, 7.0 \mathrm{~Hz}, 2 \mathrm{H}), 2.10(\mathrm{~s}, 1 \mathrm{H}), 1.54(\mathrm{~m}, 2 \mathrm{H}), 1.45(\mathrm{~m}, 2 \mathrm{H}), 0.94(\mathrm{t}, J=7.3 \mathrm{~Hz}, 3 \mathrm{H})$ ppm; ${ }^{13} \mathrm{C}\left\{{ }^{1} \mathrm{H}\right\} \mathrm{NMR}\left(\mathrm{CDCl}_{3}, 125 \mathrm{MHz}\right): \delta 143.7,140.3,127.3,109.4,86.3,79.7,57.7$, 30.8, 22.2, 18.6, and $13.8 \mathrm{ppm}$; IR (neat) 3368, 2958, 2933, 2873, 2284, 2227, 1595, $1503,1466,1380,1313,1160,1022,954,875,753 \mathrm{~cm}^{-1}$. HRMS-CI $\mathrm{m} / z 178.0986\left[\mathrm{M}^{+}\right.$; calcd for $\mathrm{C}_{11} \mathrm{H}_{14} \mathrm{O}_{2}$ : 178.0994].

\section{2-Substituted 3-Furfurals}

\section{General Procedure B: Oxidative Rearrangement of Furyl Alcohols.}

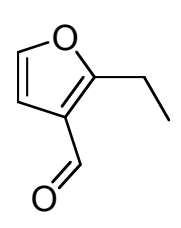

2-Ethyl-furan-3-carboxaldehyde (3a). A $10 \mathrm{~mL}$ flask was charged with 1-furan-3-yl-propan-1-ol (126 mg, $1.0 \mathrm{mmol})$ and THF $(4 \mathrm{~mL})$ and $\mathrm{H}_{2} \mathrm{O}(1$ $\mathrm{mL}$ ). NBS (178 $\mathrm{mg}, 1.0 \mathrm{mmol})$ was added to the mixture in two portions (10 $\mathrm{m}$ apart) at ambient temperature. The reaction mixture initially turned a yellow color, later fading to near colorless. The reaction was monitored by TLC, and the formation of a new, non-polar, UV active spot was observed. Upon completion (approximately $4 \mathrm{~h}$ ), 
the reaction mixture was treated with $\mathrm{HCl}_{(\mathrm{aq})}(1 \mathrm{~mL}, 1.0 \mathrm{M}, 1 \mathrm{mmol})$. After stirring for $10 \mathrm{~m}$, the reaction mixture was extracted with $\mathrm{Et}_{2} \mathrm{O}(3 \mathrm{X} 10 \mathrm{~mL})$. The combined organic layers were washed with brine, dried over $\mathrm{MgSO}_{4}$, and filtered. The filtrate was concentrated in vacuo, and the residue was chromatographed on silica $\left(5 \% \mathrm{Et}_{2} \mathrm{O}\right.$ in pentane) to afford the title compound as a yellow oil in $71 \%$ yield ( $88 \mathrm{mg}, 0.71 \mathrm{mmol}$ ).

${ }^{1} \mathrm{H} \mathrm{NMR}\left(\mathrm{CDCl}_{3}, 500 \mathrm{MHz}\right) \delta 9.96(\mathrm{~s}, 1 \mathrm{H}), 7.31(\mathrm{~d}, J=1.9 \mathrm{~Hz}, 1 \mathrm{H}), 6.68(\mathrm{~d}, J=1.9 \mathrm{~Hz}$, $1 \mathrm{H}), 2.97(\mathrm{q}, J=7.8 \mathrm{~Hz}, 2 \mathrm{H}), 1.30(\mathrm{t}, J=7.5 \mathrm{~Hz}, 3 \mathrm{H}) \mathrm{ppm} ;{ }^{13} \mathrm{C}\left\{{ }^{1} \mathrm{H}\right\} \mathrm{NMR}\left(\mathrm{CDCl}_{3}, 125\right.$ MHz): $\delta 184.8,145.0,142.1,121.9,108.1,20.6$, and 12.8 ppm; IR (neat) 2979, 2878, $2743,1770,1683,1575,1122,974$, and $753 \mathrm{~cm}^{-1}$. HRMS-CI $\mathrm{m} / z 124.0522\left[\mathrm{M}^{+}\right.$; calcd for $\mathrm{C}_{7} \mathrm{H}_{8} \mathrm{O}_{2}:$ : 124.0524].

\section{General Procedure C: One-Pot Addition / Oxidative Rearrangement of 3-Furfural.}

2-Ethyl-furan-3-carboxaldehyde (3a). Under a nitrogen atmosphere, a dry $25 \mathrm{~mL}$ flask was charged with 3-furfural $(84 \mu \mathrm{L}, 1.0 \mathrm{mmol})$ and dry THF $(1.0 \mathrm{~mL})$ and cooled to $0{ }^{\circ} \mathrm{C}$. A solution of ethyl magnesium bromide $(0.5 \mathrm{~mL}, 3.0 \mathrm{M}$ solution in ether) was added dropwise. The reaction mixture was stirred at $0{ }^{\circ} \mathrm{C}$ for $3 \mathrm{~h}$. When the addition was complete by $\mathrm{TLC}, \mathrm{HCl}_{\text {(aq) }}(1.5 \mathrm{~mL}, 1.0 \mathrm{M}, 1.5 \mathrm{mmol})$ was added. THF $(7 \mathrm{~mL})$, water ( $0.5 \mathrm{~mL})$, and NBS (178 $\mathrm{mg}, 1.0 \mathrm{mmol}$, in two portions) were then added sequentially at ambient temperature. When the rearrangement was complete by TLC, the reaction mixture was quenched with saturated aqueous $\mathrm{NH}_{4} \mathrm{Cl}$ and extracted with $\mathrm{Et}_{2} \mathrm{O}(3 \mathrm{X} 10$ $\mathrm{mL}$ ). The combined organic layers were washed with brine, dried over $\mathrm{MgSO}_{4}$, and filtered. The filtrate was concentrated in vacuo, and the residue was chromatographed on silica (5\% $\mathrm{Et}_{2} \mathrm{O}$ in pentane) to afford the title compound as a yellow oil in $70 \%$ yield ( 86 
$\mathrm{mg}, 0.70 \mathrm{mmol})$. The spectra matched that from the oxidative rearrangement.

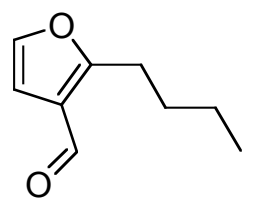

2-Butyl-furan-3-carboxaldehyde (3b). This compound was prepared by General Procedure B using 1-furan-3-yl-pentan-1-ol (154 mg, 1.0 mmol), NBS (178 mg, $1.0 \mathrm{mmol})$, THF $(4.0 \mathrm{~mL})$ and $\mathrm{H}_{2} \mathrm{O}(1.0 \mathrm{~mL})$. After the usual workup, the crude product was purified by column chromatography on deactivated silica $(0-10 \%$ EtOAc in hexanes) to afford the title compound as a yellow oil in $80 \%$ yield (100 mg, $0.8 \mathrm{mmol}) .{ }^{1} \mathrm{H}$ NMR $\left(\mathrm{C}_{6} \mathrm{D}_{6}, 360 \mathrm{MHz}\right) \delta 9.76(\mathrm{~s}, 1 \mathrm{H}), 6.73(\mathrm{~d}, J=1.5 \mathrm{~Hz}$, $1 \mathrm{H}), 6.51(\mathrm{~d}, J=1.8 \mathrm{~Hz}, 1 \mathrm{H}), 2.46(\mathrm{t}, J=7.4 \mathrm{~Hz}, 2 \mathrm{H}), 1.35(\mathrm{~m}, 2 \mathrm{H}), 1.03(\mathrm{~m}, 2 \mathrm{H}), 0.70$ (t, $J=7.3 \mathrm{~Hz}, 3 \mathrm{H}) \mathrm{ppm} ;{ }^{13} \mathrm{C}\left\{{ }^{1} \mathrm{H}\right\} \mathrm{NMR}\left(\mathrm{C}_{6} \mathrm{D}_{6}, 90 \mathrm{MHz}\right): \delta$ 184.1, 145.9, 142.2, 131.0, 108.8, 30.8, 26.8, 22.6, and $14.1 \mathrm{ppm}$; IR (neat) 3126, 2960, 2934, 2874, 2741, 1782, $1683,1575,1424,1125,1030,750 \mathrm{~cm}^{-1}$. HRMS-CI $\mathrm{m} / z 152.0844\left[\mathrm{M}^{+}\right.$; calcd for $\left.\mathrm{C}_{9} \mathrm{H}_{12} \mathrm{O}_{2}: 152.0837\right]$.

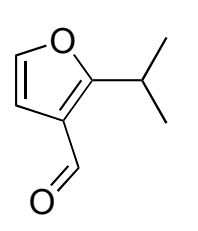

2-Isopropyl-furan-3-carboxaldehyde (3c). This compound was prepared by General Procedure B using 1-furan-3-yl-2-methyl-propan-1-ol (140 mg, 1.0 mmol), NBS (178 mg, $1.0 \mathrm{mmol})$, THF $(4.0 \mathrm{~mL})$ and $\mathrm{H}_{2} \mathrm{O}(1.0 \mathrm{~mL})$. After the usual workup, the crude product was purified by column chromatography on deactivated silica (0-10\% EtOAc in hexanes) to afford the title compound as a yellow oil in 70\% yield (97 mg, $0.7 \mathrm{mmol}) .{ }^{1} \mathrm{H} \mathrm{NMR}\left(\mathrm{CDCl}_{3}, 500 \mathrm{MHz}\right) \delta 10.01(\mathrm{~s}, 1 \mathrm{H}), 7.32(\mathrm{~d}, J$ $=1.9 \mathrm{~Hz}, 1 \mathrm{H}), 6.70(\mathrm{~d}, J=1.9 \mathrm{~Hz}, 1 \mathrm{H}), 3.54$ (septet, $J=7.0 \mathrm{~Hz}, 1 \mathrm{H}), 1.36(\mathrm{~d}, J=7.0 \mathrm{~Hz}$, 6H) ppm; ${ }^{13} \mathrm{C}\left\{{ }^{1} \mathrm{H}\right\}$ NMR $\left(\mathrm{CDCl}_{3}, 125 \mathrm{MHz}\right): \delta 184.8,145.4,142.0,121.1,108.2,27.4$, and $21.5 \mathrm{ppm}$; IR (neat) 2968, 2933, 2873, 2742, 1775, 1683, 1575, 1124, and $759 \mathrm{~cm}^{-1}$. 
HRMS-CI $m / z 139.0757$ [(MH) ${ }^{+}$; calcd for $\left.\mathrm{C}_{8} \mathrm{H}_{11} \mathrm{O}_{2}: 139.0759\right]$.

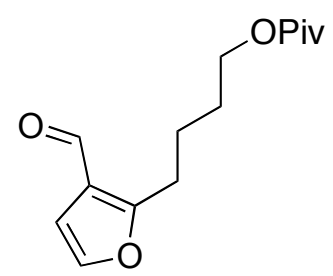

\section{2,2- Dimethyl propionic acid 4-(3-formyl-furan-2-yl)-butyl ester}

(3d). This compound was prepared by General Procedure B using 2,2-dimethyl propionic acid ester- furan-3-yl-butanol (254 mg, 1.0 mmol), NBS (178 mg, $1.0 \mathrm{mmol})$, THF $(4.0 \mathrm{~mL})$ and $\mathrm{H}_{2} \mathrm{O}(1.0 \mathrm{~mL})$. After the usual workup, the crude product was purified by column chromatography on deactivated silica $(0-10 \%$ EtOAc in hexanes) to afford the title compound as a yellow oil in $80 \%$ yield (201 mg, $0.8 \mathrm{mmol}) .{ }^{1} \mathrm{H} \mathrm{NMR}\left(\mathrm{CDCl}_{3}, 500 \mathrm{MHz}\right) \delta 9.95(\mathrm{~s}, 1 \mathrm{H}), 7.33(\mathrm{~s}, 1 \mathrm{H}), 6.71(\mathrm{~s}, 1 \mathrm{H})$, $4.09(\mathrm{t}, J=6.5 \mathrm{~Hz}, 2 \mathrm{H}), 3.02(\mathrm{t}, J=8.5 \mathrm{~Hz}, 2 \mathrm{H}), 1.82(\mathrm{~m}, 1 \mathrm{H}), 1.71(\mathrm{~m}, 1 \mathrm{H})$ and $1.21(\mathrm{~s}$, 9H) ppm; ${ }^{13} \mathrm{C}\left\{{ }^{1} \mathrm{H}\right\}$ NMR $\left(\mathrm{CDCl}_{3}, 125 \mathrm{MHz}\right): \delta$ 184.7, 178.5, 165.0, 142.1,122.5, 108.1, 63.6, 36.6, 29.7, 28.4, 27.1, and $24.7 \mathrm{ppm}$; IR (neat) 3412, 2962, 2360, 1725, 1480, 1458, $1398,1285,1158,1035$, and $771 \mathrm{~cm}^{-1}$. HRMS-CI $m / z 252.1341\left[\mathrm{M}^{+}\right.$; calcd for $\mathrm{C}_{14} \mathrm{H}_{20} \mathrm{O}_{4}$ : 252.1362].

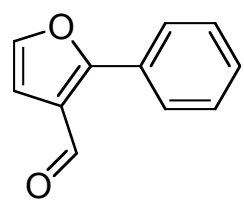

2-Phenyl-furan-3-carboxaldehyde (3e). This compound was prepared by General Procedure B using furan-3-yl-phenyl-methanol (174 mg, 1.0 mmol), NBS (178 mg, $1.0 \mathrm{mmol})$, THF $(4.0 \mathrm{~mL})$ and $\mathrm{H}_{2} \mathrm{O}(1.0 \mathrm{~mL})$. After the usual workup, the crude product was purified by column chromatography on deactivated silica ( $0-10 \%$ EtOAc in hexanes) to afford the title compound as a yellow oil in $80 \%$ yield (124 mg, $0.72 \mathrm{mmol}) .{ }^{1} \mathrm{H}$ NMR $\left(\mathrm{CDCl}_{3}, 500 \mathrm{MHz}\right) \delta 10.15(\mathrm{~s}, 1 \mathrm{H}), 7.77-$ $7.75(\mathrm{~m}, 2 \mathrm{H}), 7.55-7.51(\mathrm{~m}, 3 \mathrm{H}), 7.50(\mathrm{~d}, J=1.8 \mathrm{~Hz}, 1 \mathrm{H}), 6.93(\mathrm{~d}, J=1.9 \mathrm{~Hz}, 1 \mathrm{H}) \mathrm{ppm}$; ${ }^{13} \mathrm{C}\left\{{ }^{1} \mathrm{H}\right\}$ NMR $\left(\mathrm{CDCl}_{3}, 75 \mathrm{MHz}\right): \delta 185.9,161.8,143.0,130.4,129.2,129.1,128.3$, 
123.2, and 109.7 ppm; IR (neat) 3126, 2849, 2752, 1676, 1488, 1269, 1145, 771, and $693 \mathrm{~cm}^{-1}$. HRMS-CI $m / z 172.0516\left[\mathrm{M}^{+}\right.$; calcd for $\left.\mathrm{C}_{11} \mathrm{H}_{8} \mathrm{O}_{2}: 172.0524\right]$.

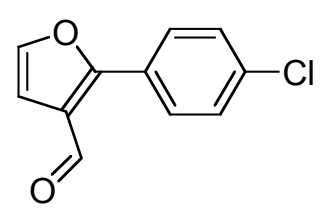

2-(4-Chloro-phenyl)-furan-carboxaldehyde (3f). This compound was prepared by General Procedure B using 2-(4-chloro-phenyl)furan-3-yl-methanol (208 mg, $1.0 \mathrm{mmol})$, NBS (178 mg, $1.0 \mathrm{mmol})$, THF (4.0 mL) and $\mathrm{H}_{2} \mathrm{O}(1.0 \mathrm{~mL})$. After the usual workup, the crude product was purified by column chromatography on deactivated silica $(0-10 \%$ EtOAc in hexanes) to afford the title compound as a yellow oil in $72 \%$ yield $(148 \mathrm{mg}, 0.72 \mathrm{mmol}) .{ }^{1} \mathrm{H}$ NMR $\left(\mathrm{CDCl}_{3}, 300\right.$ MHz) $\delta 10.10(\mathrm{~s}, 1 \mathrm{H}), 7.73(\mathrm{~d}, J=9.0 \mathrm{~Hz}, 2 \mathrm{H}), 7.47(\mathrm{~m}, 3 \mathrm{H}), 6.91(\mathrm{~s}, 1 \mathrm{H}) \mathrm{ppm} ;{ }^{13} \mathrm{C}\left\{{ }^{1} \mathrm{H}\right\}$ NMR $\left(\mathrm{CDCl}_{3}, 75 \mathrm{MHz}\right): \delta 186.7,144.2,137.6,130.6,130.5,130.4,130.3,128.6,124.3$, 112.1, 111.1 ppm; IR (neat) 3425, 3125, 2849, 1904, 1776, 1720, 1674, 1602, 1577, $1548,1517,1483,1419,1406,1270,1181,1144,1094,1041,1013,930,889,883,775$, 757, and $721 \mathrm{~cm}^{-1}$. HRMS-CI $\mathrm{m} / z 206.0135$ [M+calcd for $\left.\mathrm{C}_{11} \mathrm{H}_{7} \mathrm{O}_{2}: 206.0134\right]$.

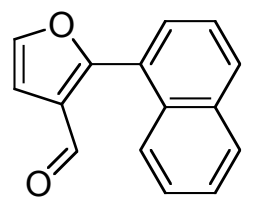

2-Naphthalen-1-yl-furan-3-carbaldehyde (3g). This compound was prepared by General Procedure B using 2-(1-napthal)-furan-3-ylmethanol (224 mg, $1.0 \mathrm{mmol})$, NBS (178 mg, $1.0 \mathrm{mmol})$, THF (4.0 mL) and $\mathrm{H}_{2} \mathrm{O}(1.0 \mathrm{~mL})$. After the usual workup, the crude product was purified by column chromatography on deactivated silica $(0-10 \%$ EtOAc in hexanes) to afford the title compound as a yellow oil in $75 \%$ yield $(167 \mathrm{mg}, 0.75 \mathrm{mmol}) .{ }^{1} \mathrm{H} \mathrm{NMR}\left(\mathrm{CDCl}_{3}, 500\right.$ MHz) $\delta 9.85(\mathrm{~s}, 1 \mathrm{H}), 7.98(\mathrm{~m}, 3 \mathrm{H}), 7.59(\mathrm{~m}, 5 \mathrm{H})$ and $7.05(\mathrm{~d}, J=2.0 \mathrm{~Hz}, 1 \mathrm{H}) \mathrm{ppm}$; ${ }^{13} \mathrm{C}\left\{{ }^{1} \mathrm{H}\right\}$ NMR $\left(\mathrm{CDCl}_{3}, 125 \mathrm{MHz}\right): \delta 186.0,162.7,143.5,133.8,131.9,131.1,130.0$, 
128.6, 127.4, 126.7, 125.7, 125.3, 125.06, 125.0 and $108.5 \mathrm{ppm}$; IR (neat) 3337, $3150,3123,3050,2836,2741,2252,1933,1820,1777,1676,1588,1566,1518,1505$, $1461,1422,1380,1339,1270,1248,1214,1189,1142,1114,1063,1046,1030,1020$ 973, 958, 891, 803, 776, 751, and $704 \mathrm{~cm}^{-1}$. HRMS-CI $\mathrm{m} / z 222.0667\left[\mathrm{M}^{+}\right.$; calcd for $\left.\mathrm{C}_{15} \mathrm{H}_{10} \mathrm{O}_{2}: 222.0680\right]$.

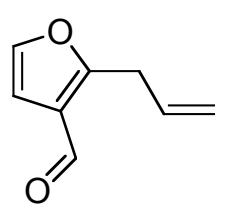

2-Isopropenyl-furan-3-carbaldehyde (3h). This compound was prepared by General Procedure B using 2-allyl-furan-3-yl-methanol (138 mg, 1.0 mmol), NBS (178 mg, $1.0 \mathrm{mmol})$, THF (4.0 mL) and $\mathrm{H}_{2} \mathrm{O}(1.0 \mathrm{~mL})$. After the usual workup, the crude product was purified by column chromatography on deactivated silica ( $0-10 \%$ EtOAc in hexanes) to afford the title compound as a yellow oil in $73 \%$ yield $(99 \mathrm{mg}, 0.73 \mathrm{mmol}) ;{ }^{1} \mathrm{H}$ NMR $\left(\mathrm{CDCl}_{3}, 360 \mathrm{MHz}\right) \delta 10.00$ (s, $\left.1 \mathrm{H}\right), 7.36$ (d, $J$ $=1.8 \mathrm{~Hz}, 1 \mathrm{H}), 6.74(\mathrm{~d}, J=1.9 \mathrm{~Hz}, 1 \mathrm{H}), 5.97(\mathrm{~m}, 1 \mathrm{H}), 5.22(\mathrm{~s}, 1 \mathrm{H}), 5.18(\mathrm{~d}, J=6.0 \mathrm{~Hz}$, 1H), $3.75(\mathrm{~d}, J=6.4 \mathrm{~Hz}, 2 \mathrm{H}) \mathrm{ppm} ;{ }^{13} \mathrm{C}\left\{{ }^{1} \mathrm{H}\right\} \mathrm{NMR}\left(\mathrm{CDCl}_{3}, 75 \mathrm{MHz}\right): \delta$ 185.1, 163.1, 146.2, 142.8, 132.6, 118.5, 108.6, and $31.8 \mathrm{ppm}$; IR (neat) $3365,2950,2358,1714$, and $1180 \mathrm{~cm}^{-1}$. HRMS-CI $\mathrm{m} / z 137.0605\left[\mathrm{MH}^{+}\right.$; calcd for $\left.\mathrm{C}_{8} \mathrm{H}_{9} \mathrm{O}_{2}: 137.0597\right]$.

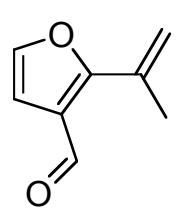

2-Isopropenyl-furan-3-carbaldehyde (3i). This compound was prepared by

General Procedure B using 1-furan-3-yl-2-methyl-prop-2-en-1-ol (138 mg, $1.0 \mathrm{mmol})$, NBS $(178 \mathrm{mg}, 1.0 \mathrm{mmol})$, THF $(4.0 \mathrm{~mL})$ and $\mathrm{H}_{2} \mathrm{O}(1.0 \mathrm{~mL})$. After the usual workup, the crude product was purified by column chromatography on deactivated silica ( $0-10 \%$ EtOAc in hexanes) to afford the title compound as a yellow oil in $72 \%$ yield $(98 \mathrm{mg}, 0.72 \mathrm{mmol}) .{ }^{1} \mathrm{H} \mathrm{NMR}\left(\mathrm{CDCl}_{3}, 300 \mathrm{MHz}\right) \delta 10.10(\mathrm{~s}, 1 \mathrm{H}), 7.36(\mathrm{~d}$, 


$$
J=2.0 \mathrm{~Hz}, 1 \mathrm{H}), 6.83(\mathrm{~d}, J=2.0 \mathrm{~Hz}, 1 \mathrm{H}), 5.63(\mathrm{~s}, 1 \mathrm{H}), 5.45(\mathrm{~s}, 1 \mathrm{H}), 2.23(\mathrm{~s}, 1 \mathrm{H})
$$

ppm; ${ }^{13} \mathrm{C}\left\{{ }^{1} \mathrm{H}\right\}$ NMR $\left(\mathrm{CDCl}_{3}, 125 \mathrm{MHz}\right): \delta$ 186.5, 139.2, 129.8, 128.6, 127.6, 119.3, 108.9, and $21.3 \mathrm{ppm}$; IR (neat) 3393, 2926, 2357, 1733, 1376, 1259, 1157, 803, and 748 $\mathrm{cm}^{-1}$. HRMS-CI $m / z 136.0530\left[\mathrm{M}^{+}\right.$; calcd for $\left.\mathrm{C}_{8} \mathrm{H}_{8} \mathrm{O}_{2}: 136.0524\right]$.

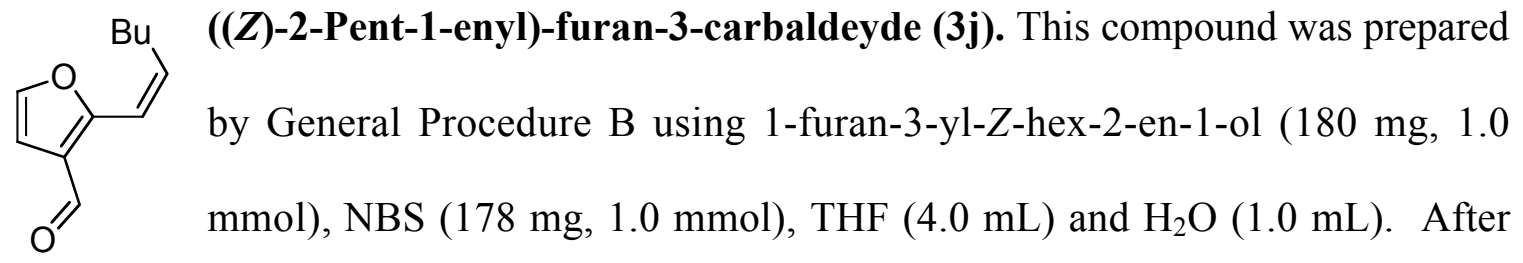

the usual workup, the crude product was purified by column chromatography on deactivated silica (0-10\% EtOAc in hexanes) to afford the title compound as a yellow oil in 70\% yield (125 mg, $0.70 \mathrm{mmol}) ;{ }^{1} \mathrm{H}$ NMR $\left(\mathrm{CDCl}_{3}, 500 \mathrm{MHz}\right) \delta 10.05(\mathrm{~s}, 1 \mathrm{H}), 7.42(\mathrm{~d}$, $J=1.5 \mathrm{~Hz}, 1 \mathrm{H}), 6.79(\mathrm{~d}, J=1.5 \mathrm{~Hz}, 1 \mathrm{H}), 6.66(\mathrm{~d}, J=10.0 \mathrm{~Hz}, 1 \mathrm{H}), 6.00(\mathrm{~m}, 1 \mathrm{H}), 2.64$ $(\mathrm{q}, J=5.0 \mathrm{~Hz}, 2 \mathrm{H}), 1.53(\mathrm{~m}, 2 \mathrm{H}), 1.43(\mathrm{~m}, 2 \mathrm{H}), 0.96(\mathrm{t}, J=5.0 \mathrm{~Hz}, 3 \mathrm{H}) \mathrm{ppm} ;{ }^{13} \mathrm{C}\left\{{ }^{1} \mathrm{H}\right\}$ NMR $\left(\mathrm{CDCl}_{3}, 125 \mathrm{MHz}\right): \delta 184.5,160.1,142.4,119.6,119.4,113.7,108.6,31.6,29.7$, 22.4, and $13.9 \mathrm{ppm}$; IR (neat) 2926, 2359, 2339, 1676, 1641, 1549, 1506, 1433, 1127, 894, and $741 \mathrm{~cm}^{-1}$. HRMS-CI $m / z 178.0999$ [M+; calcd for $\mathrm{C}_{11} \mathrm{H}_{14} \mathrm{O}_{2}$ : 178.0994].

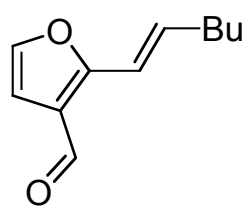

((E)-2-Pent-1-enyl)-furan-3-carbaldeyde (3k). This compound was prepared by General Procedure B using 1-furan-3-yl-E-hex-2-en-1-ol (180 mg, $1.0 \mathrm{mmol})$, NBS (178 mg, $1.0 \mathrm{mmol})$, THF (4.0 mL) and $\mathrm{H}_{2} \mathrm{O}$ $(1.0 \mathrm{~mL})$. After the usual workup, the crude product was purified by column chromatography on deactivated silica $(0-10 \%$ EtOAc in hexanes) to afford the title compound as a yellow oil in $70 \%$ yield $(125 \mathrm{mg}, 0.70 \mathrm{mmol}) .{ }^{1} \mathrm{H}$ NMR $\left(\mathrm{CDCl}_{3}, 500\right.$ 
MHz) $\delta 9.95(\mathrm{~s}, 1 \mathrm{H}), 7.23(\mathrm{~d}, J=1.5 \mathrm{~Hz}, 1 \mathrm{H}), 6.66(\mathrm{~d}, J=1.5 \mathrm{~Hz}, 1 \mathrm{H}), 6.63(\mathrm{~d}, J=$ $20.0 \mathrm{~Hz}, 1 \mathrm{H}), 6.55(\mathrm{~m}, 1 \mathrm{H}), 2.22(\mathrm{q}, J=5.5 \mathrm{~Hz}, 2 \mathrm{H}), 1.44(\mathrm{~m}, 2 \mathrm{H}), 1.40(\mathrm{~m}, 2 \mathrm{H})$, and $0.88(\mathrm{t}, J=10.0 \mathrm{~Hz}, 3 \mathrm{H}) \mathrm{ppm} ;{ }^{13} \mathrm{C}\left\{{ }^{1} \mathrm{H}\right\} \mathrm{NMR}\left(\mathrm{CDCl}_{3}, 125 \mathrm{MHz}\right): \delta 184.6,159.8,142.4$, 139.0, 121.5, 115.9, 109.2, 33.1, 31.2, and $14.1 \mathrm{ppm}$; IR (neat) 3152, 3126, 2958, 2929, $2872,2859,2777,2739,1780,1755,1723,1676,1652,1624,1553,1509,1465,1428$, $1395,1379,1340,1160,1130,1049,1034,965,929,895,873,808,781,738,686$, and $600 \mathrm{~cm}^{-1}$. HRMS-CI $\mathrm{m} / z$ 179.1066 [MH'; calcd for $\left.\mathrm{C}_{11} \mathrm{H}_{15} \mathrm{O}_{2}: 179.1072\right]$.

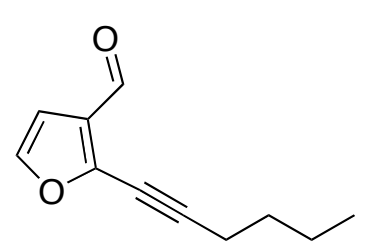

2-Hex-1-ynyl-furan-3-carbaldehyde (31). This compound was prepared by General Procedure B using 1-furan-3-yl-hept-2-yn-1ol (482.6 mg, $2.71 \mathrm{mmol})$, THF (10.8 mL), $\mathrm{H}_{2} \mathrm{O}(2.7 \mathrm{~mL})$ and NBS (482.4 $\mathrm{mg}, 2.71 \mathrm{mmol}$ ). The reaction was stirred at $\mathrm{rt}$ for $14 \mathrm{~h}$. After the usual workup, the residue was purified by column chromatography on silica $(2.5-10 \%$ EtOAc in hexanes) to afford the title compound as a colorless to slightly yellow oil in $79 \%$ yield (377.5 mg, $2.14 \mathrm{mmol}) .{ }^{1} \mathrm{H} \mathrm{NMR}\left(\mathrm{CDCl}_{3}, 500 \mathrm{MHz}\right) \delta 10.00(\mathrm{~s}, 1 \mathrm{H}), 7.34(\mathrm{~d}, J=1.7 \mathrm{~Hz}$, $1 \mathrm{H}), 6.75(\mathrm{~d}, J=1.7 \mathrm{~Hz}, 1 \mathrm{H}), 2.55(\mathrm{t}, J=7.1 \mathrm{~Hz}, 2 \mathrm{H}), 1.66(\mathrm{~m}, 2 \mathrm{H}), 1.51(\mathrm{~m}, 2 \mathrm{H}), 0.98$ (t, $J=7.3 \mathrm{~Hz}, 3 \mathrm{H}) \mathrm{ppm} ;{ }^{13} \mathrm{C}\left\{{ }^{1} \mathrm{H}\right\} \mathrm{NMR}\left(\mathrm{CDCl}_{3}, 125 \mathrm{MHz}\right): \delta 185.1,146.1,134.9,128.9$, 107.7, 101.6, 68.9, 30.3, 22.2, 19.6, 13.7 ppm; IR (neat) 3129, 2960, 2934, 2873, 2830, $2739,2830,2739,2231,1686,1569,1505,1427,1254,1134,1068,892,763 \mathrm{~cm}^{-1}$. HRMS-CI $m / z$ 176.0836 [M" ; calcd for $\mathrm{C}_{11} \mathrm{H}_{12} \mathrm{O}_{2}:$ 176.0837].

2-Hex-1-ynyl-furan-3-carbaldehyde (3I). For the one-pot method, This compound was prepared by General Proceedure $\mathrm{C}$ using n-BuLi $(0.565 \mu \mathrm{L}, 2.48 \mathrm{M}$ in hexanes), 1- 
hexyne $(173.6 \mu \mathrm{L}, 1.5 \mathrm{mmol})$, dry THF $(4 \mathrm{~mL})$ and 3-furfural $(84 \mu \mathrm{L}, 1.0 \mathrm{mmol})$ were used. After the addition step was complete (by TLC), $\mathrm{HCl}_{(\mathrm{aq})}\left(0.7 \mathrm{~mL}, 2 \mathrm{~N}\right.$ in $\mathrm{H}_{2} \mathrm{O}$ ) and $\mathrm{DI} \mathrm{H}_{2} \mathrm{O}(0.3 \mathrm{~mL})$ were added followed by NBS $(178 \mathrm{mg}, 1 \mathrm{mmol})$ in two portions (2 $\mathrm{x} 89 \mathrm{mg}$ ) and stirred at $\mathrm{rt}$ for $14 \mathrm{~h}$. The quench, workup and purification were identical to the two-step procedure. The title compound was afforded in $65 \%$ yield $(113.8 \mathrm{mg}, 0.65$ mmol).

\section{$\underline{\text { Deuterium Mechanistic Study }}$}<smiles>[2H]c1occc1C=O</smiles>

2-Deutero-3-furfural (1-d $\left.\mathbf{d}_{\mathbf{1}}\right)$. To a solution of N, N, N'-trimethylethylene diamine $(0.466 \mathrm{~mL}, 3.6 \mathrm{mmol})$ in THF $(10 \mathrm{~mL})$ at $-78^{\circ} \mathrm{C}$ was added $n$-BuLi (1.32 mL, 2.5 M in hexanes). ${ }^{8}$ After $15 \mathrm{~m}$, freshly distilled 3-furfural $(0.25 \mathrm{~mL}$, $3 \mathrm{mmol})$ was added and the mixture was stirred for another $15 \mathrm{~m}$. $n$-BuLi (6 mmol) was added and stirred for $2 \mathrm{~h}$ at $-78^{\circ} \mathrm{C}$. Quenching with deuterium oxide (326 $\left.\mu \mathrm{L}, 18 \mathrm{mmol}\right)$ at $-78{ }^{\circ} \mathrm{C}$. Drying over $\mathrm{MgSO}_{4}$, filtration, and concentration with a rotary evaporator followed by a quick flash column on silica (5\% $\mathrm{Et}_{2} \mathrm{O} /$ pentane) yielded 2-deutero-3furaldehyde as a clear to slightly yellow liquid with $80 \%$ deuterium incorporation by ${ }^{1} \mathrm{H}$ NMR. ${ }^{9}$ Due to its sensitive nature, this material was immediately carried though to the next step. ${ }^{10}$

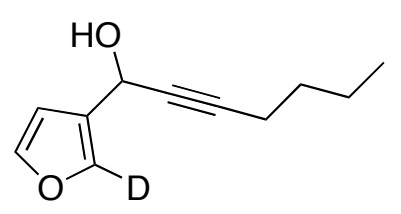

2-Deutero-1-furan-3-yl-hept-2-yn-1-ol (2l- $\left.\boldsymbol{d}_{1}\right)$. To a solution of 1-hexyne $(174 \mu \mathrm{L}, 1.5 \mathrm{mmol})$ in THF $(4 \mathrm{~mL})$ was added $\mathrm{n}$-BuLi $\left(0.56 \mathrm{~mL}, 1.4 \mathrm{mmol}, 2.5 \mathrm{M}\right.$ in hexanes) dropwise at $-78^{\circ} \mathrm{C}$ and stirred for $40 \mathrm{~min}$. Warming to $0{ }^{\circ} \mathrm{C}$, 2-deutero-3-furfural $(72 \mathrm{mg}, 0.74 \mathrm{mmol})$ in THF (2 
$\mathrm{X} 1 \mathrm{~mL}$ ) was added dropwise via cannula, and was allowed to warm to rt over 1-2 hr. The reaction was quenched with $\mathrm{H}_{2} \mathrm{O}$ and $\mathrm{HCl}\left(2 \mathrm{~N}\right.$ in $\left.\mathrm{H}_{2} \mathrm{O}\right)$, and $\mathrm{Et}_{2} \mathrm{O}(2 \mathrm{~mL})$ was added. After the organic and aqueous layers were separated, the aqueous layer was extracted with $\mathrm{Et}_{2} \mathrm{O}(3 \times 10 \mathrm{~mL})$. The combined organic layers were washed with sat. $\mathrm{NaHCO}_{3}$, brine, dried over $\mathrm{MgSO}_{4}$, filtered, and the filtrate was concentrated in vacuo. The residue was purified by column chromatography on silica (5-20\% EtOAc in hexanes) to afford the title compound as a colorless oil in $92 \%$ yield $(122 \mathrm{mg}, 0.68 \mathrm{mmol}) .{ }^{1} \mathrm{H}$ NMR $\left(\mathrm{CDCl}_{3}, 500 \mathrm{MHz}\right) \delta 7.53$ (s, 0.2H/0.8D), 7.41 (s, 1H), 6.52 (s, 1H), $5.40(\mathrm{~s}, 1 \mathrm{H})$, $2.28(\mathrm{dt}, J=1.7,7.0 \mathrm{~Hz}, 2 \mathrm{H}), 2.03(\mathrm{~s}, 1 \mathrm{H}), 1.54(\mathrm{~m}, 2 \mathrm{H}), 1.45(\mathrm{~m}, 2 \mathrm{H}), 0.94(\mathrm{t}, J=7.3$ $\mathrm{Hz}, 3 \mathrm{H}) \mathrm{ppm} ;{ }^{13} \mathrm{C}\left\{{ }^{1} \mathrm{H}\right\} \mathrm{NMR}\left(\mathrm{CDCl}_{3}, 125 \mathrm{MHz}\right): \delta 143.7,140.3,127.1,109.4,86.3,79.7$, 57.7, 30.8, 22.2, 18.6, 13.8 ppm; IR (neat) 3360, 2959, 2934, 2873, 2285, 2226, 1593, $1489,1465,1311,1261,1158,1005,820,740 \mathrm{~cm}^{-1}$. HRMS-CI $m / z 179.1056\left[\mathrm{M}^{+}\right.$; calcd for $\mathrm{C}_{11} \mathrm{H}_{13} \mathrm{DO}_{2}$ : 179.1055].

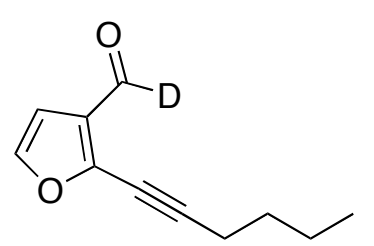

2-Hex-1-ynyl-furan-3-carbaldehyde- $d_{1}\left(3 I-d_{1}\right)$. This compound was prepared by General Procedure B using 2-deutero-1-furan-3yl-hept-2-yn-1-ol (65.5 mg, $0.365 \mathrm{mmol})$, THF (1.5 mL), $\mathrm{H}_{2} \mathrm{O}$ (0.37 mL), and NBS (65 mg, $0.365 \mathrm{mmol})$. The reaction was stirred at $\mathrm{rt}$ for $14 \mathrm{~h}$. After the usual workup, the resulting residue was purified by column chromatography on silica (2.5-10\% EtOAc in hexanes) to afford the title compound. ${ }^{1} \mathrm{H} \mathrm{NMR}\left(\mathrm{CDCl}_{3}, 500 \mathrm{MHz}\right) \delta$ $10.0(\mathrm{~s}, 0.2 \mathrm{H} / 0.8 \mathrm{D}), 7.33(\mathrm{~d}, J=1.7 \mathrm{~Hz}, 1 \mathrm{H}), 6.74(\mathrm{~d}, J=1.6 \mathrm{~Hz}, 1 \mathrm{H}), 2.54(\mathrm{t}, J=7.1 \mathrm{~Hz}$ 2H), $1.66(\mathrm{~m}, 2 \mathrm{H}), 1.51(\mathrm{~m}, 2 \mathrm{H}), 0.97(\mathrm{t}, J=7.3 \mathrm{~Hz}, 3 \mathrm{H}) \mathrm{ppm} ;{ }^{13} \mathrm{C}\left\{{ }^{1} \mathrm{H}\right\} \mathrm{NMR}\left(\mathrm{CDCl}_{3}\right.$, $125 \mathrm{MHz}): \delta 185.1,146.1,143.9,128.8,107.7,101.6,68.9,30.2,22.2,19.5,13.7$ ppm; 
IR (neat) 3129, 2960, 2934, 2873, 2739, 2231, 2100, 1671, 1566, 1505, 1423, 1264 , 1135, 1042, 892, $759 \mathrm{~cm}^{-1}$. HRMS-CI $\mathrm{m} / z 177.0898\left[\mathrm{M}^{+}\right.$; calcd for $\mathrm{C}_{11} \mathrm{H}_{11} \mathrm{DO}_{2}$ : 177.0903].

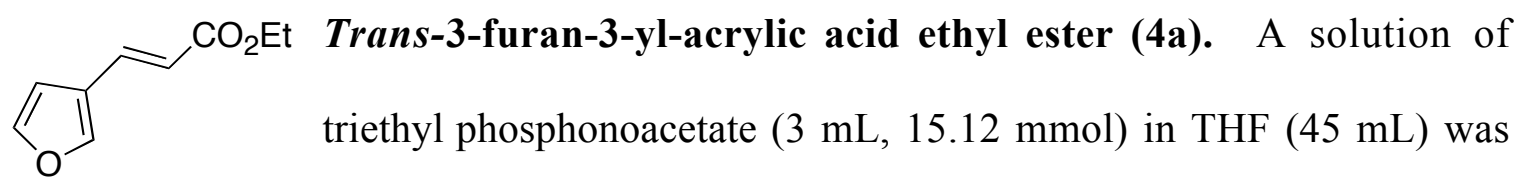
added $n$-BuLi (7.2 mL, 2.5 M in hexanes) at $0{ }^{\circ} \mathrm{C}$ and stirred for $10 \mathrm{~m}$. 3-Furfural (1.39 $\mathrm{mL}, 16.6 \mathrm{mmol}$ ) was added to the mixture dropwise and stirred for $30 \mathrm{~m}$. After the reaction mixture was quenched with sat. $\mathrm{NH}_{4} \mathrm{Cl}$, the aqueous layer was extracted with EtOAc $(3 \times 25 \mathrm{~mL})$. The combined organic layers were washed with water, brine, and dried over $\mathrm{MgSO}_{4}$, filtered, and the filtrate was concentrated in vacuo. Column chromatography on silica (0-10\% EtOAc in hexanes) afforded the title compound in $76 \%$ $(1.90 \mathrm{~g}, 11.4 \mathrm{mmol})$ as an off white to yellow solid (yellows over time with prolonged exposure to light). ${ }^{1} \mathrm{H} \mathrm{NMR}\left(\mathrm{CDCl}_{3}, 500 \mathrm{MHz}\right) \delta 7.66(\mathrm{~s}, 1 \mathrm{H}), 7.59(\mathrm{~d}, J=15.8 \mathrm{~Hz}, 1 \mathrm{H})$, $7.45(\mathrm{~s}, 1 \mathrm{H}), 6.63,1 \mathrm{H}), 6.18(\mathrm{~d}, J=15.8 \mathrm{~Hz}, 1 \mathrm{H}), 4.62(\mathrm{q}, J=7.1 \mathrm{~Hz}, 2 \mathrm{H}), 1.34(\mathrm{t}, J=$ $7.1 \mathrm{~Hz}, 3 \mathrm{H}) \mathrm{ppm} ;{ }^{13} \mathrm{C}\left\{{ }^{1} \mathrm{H}\right\} \mathrm{NMR}\left(\mathrm{CDCl}_{3}, 125 \mathrm{MHz}\right): \delta 167.2,144.6,144.6,134.7,122.8$, 118.3, 107.7, 60.6, and $14.5 \mathrm{ppm}$; IR (neat) 3137, 3122, 2979, 2904, 2302, 2236, 1963, $1702,1644,1368,1317,1280,1221,1191,1153,1041,1018$, and $870 \mathrm{~cm}^{-1}$. HRMS-CI $m / z$ 166.0637 $\left[\mathrm{M}^{+}\right.$; calcd for $\left.\mathrm{C}_{9} \mathrm{H}_{10} \mathrm{O}_{3}: 166.0630\right] . \mathrm{mp}=36-38{ }^{\circ} \mathrm{C}$.

\section{$\underline{\text { Enantioenriched Furyl Diol products }}$}

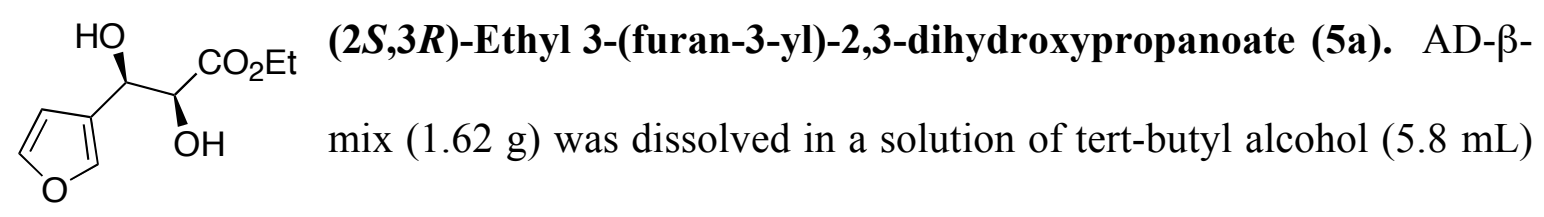


and $\mathrm{H}_{2} \mathrm{O}(5.8 \mathrm{~mL})$. Methanesulfonamide $(110 \mathrm{mg}, 1.16 \mathrm{mmol})$ was dissolved in the biphasic solution. Trans-3-furan-3-yl-acrylic acid ethyl ester was added and the mixture was stirred at rt. After $20 \mathrm{~h}$, the flask was charged with an additional $0.2 \mathrm{~g}$ of AD $\beta$-mix (as alkene starting material remained by TLC). After $8 \mathrm{~h}$, sodium sulfite (1.74 g) was added and stirred for $30 \mathrm{~min}$. DCM $(12 \mathrm{~mL})$ was added, and the phases were separated. The aqueous phase was extracted with DCM $(3 \times 6 \mathrm{~mL})$. The combined organic layers were washed with $\mathrm{KOH}(2 \mathrm{~N})$, dried over $\mathrm{MgSO}_{4}$, filtered and the filtrate was concentrated in vacuo. Column chromatography on silica (0-10\% EtOAc in hexanes) afforded the title compound in $78 \%$ yield $(181.4 \mathrm{mg}, 0.906 \mathrm{mmol})$ as a white solid. $[\alpha]_{\mathrm{D}}{ }^{20}=-3.57^{\circ}(\mathrm{c}=2.61, \mathrm{DCM}, 99 \% \mathrm{ee}) ;{ }^{1} \mathrm{H} \mathrm{NMR}\left(\mathrm{CDCl}_{3}, 500 \mathrm{MHz}\right) \delta 7.50(\mathrm{~s}, 1 \mathrm{H})$, $7.40(\mathrm{~d}, J=1.6 \mathrm{~Hz}, 1 \mathrm{H}), 6.48(\mathrm{~d}, J=1.5 \mathrm{~Hz}, 1 \mathrm{H}), 4.96(\mathrm{~s}, 1 \mathrm{H}), 4.32(\mathrm{~s}, 1 \mathrm{H}), 4.28(\mathrm{q}, J=$ $7.1 \mathrm{~Hz}, 2 \mathrm{H}), 3.50(\mathrm{~s}, 1 \mathrm{H}), 2.92(\mathrm{~s}, 1 \mathrm{H}), 1.30(\mathrm{t}, J=7.1 \mathrm{~Hz}, 3 \mathrm{H}) \mathrm{ppm} ;{ }^{13} \mathrm{C}\left\{{ }^{1} \mathrm{H}\right\} \mathrm{NMR}$ $\left(\mathrm{CDCl}_{3}, 125 \mathrm{MHz}\right): \delta 172.9,143.4,140.4,125.2,109.4,74.1,68.1,62.4$, and $14.2 \mathrm{ppm}$; IR (neat) 3434, 3148, 2985, 2937, 1733, 1634, 1504, 1391, 1371, 1282, 1221, 1159, $1118,1045,1021,950,875 \mathrm{~cm}^{-1}$. HRMS-CI $\mathrm{m} / z 199.0601\left[(\mathrm{M}-\mathrm{H})^{+}\right.$; calcd for $\mathrm{C}_{9} \mathrm{H}_{11} \mathrm{O}_{5}$ : 199.0606]. $\mathrm{mp}=49.5-50.5^{\circ} \mathrm{C}$.

General Procedure D: Sharpless Asymmetric Dihydroxylation of trans-3Vinylfurans.

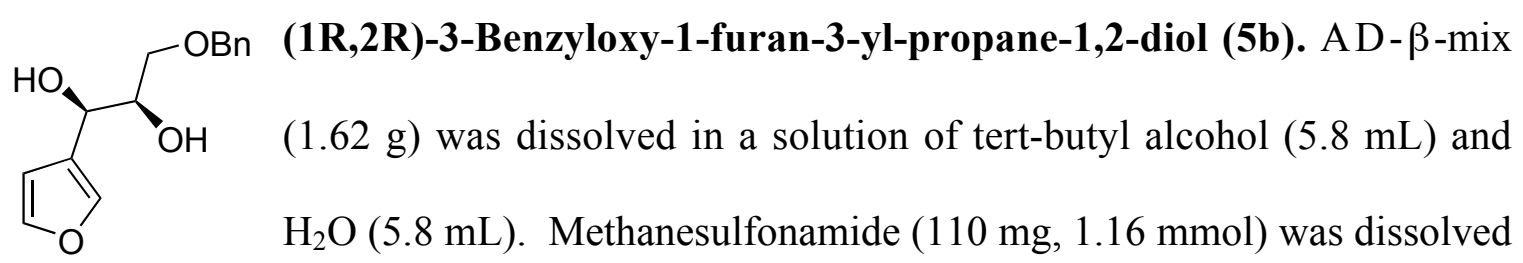

in the biphasic solution. At $0{ }^{\circ} \mathrm{C}$, trans-2-benzyloxy-1 furan-3-yl-propene (214 mg, 1.16 
mmol) was added and the mixture. After $20 \mathrm{~h}$ at $0^{\circ} \mathrm{C}$, the flask was charged with an additional AD- $\beta$-mix ( $0.2 \mathrm{~g}$ ) as olefin starting material still remained (by TLC). After $8 \mathrm{~h}$, sodium sulfite $(1.74 \mathrm{~g})$ was added and stirred for $30 \mathrm{~m}$ at $\mathrm{rt}$. DCM $(12 \mathrm{~mL})$ was added, and the phases were separated. The aqueous phase was extracted with DCM $(3 \times 6 \mathrm{~mL})$. The combined organic layers were washed with $\mathrm{KOH}(2 \mathrm{~N})$, dried over $\mathrm{MgSO}_{4}$, filtered and the filtrate was concentrated in vacuo. The crude product was purified by column chromatography on deactivated silica (20\% EtOAc in hexanes) to afford the title compound as a yellow oil in 79\% yield $(196 \mathrm{mg}, 0.79 \mathrm{mmol}) ;[\alpha]_{\mathrm{D}}{ }^{20}=-335.7(c=0.014$, $\mathrm{CHCl}_{3}, 99 \%$ ee); ${ }^{1} \mathrm{H} \mathrm{NMR}\left(\mathrm{CDCl}_{3}, 500 \mathrm{MHz}\right) \delta 7.36(\mathrm{~m}, 7 \mathrm{H}), 6.42(\mathrm{~s}, 1 \mathrm{H}), 4.72(\mathrm{~d}, J=$ $5.0 \mathrm{~Hz}, 1 \mathrm{H}), 4.58(\mathrm{~d}, J=12.0 \mathrm{~Hz}, 1 \mathrm{H}), 4.53(\mathrm{~d}, J=12.0 \mathrm{~Hz}, 1 \mathrm{H}) 3.88(\mathrm{br} \mathrm{s}, 1 \mathrm{H}), 3.60(\mathrm{~m}$, 1H), $3.53(\mathrm{~m}, 1 \mathrm{H})$, and $3.07(\mathrm{~s}, 1 \mathrm{H}) \mathrm{ppm} ;{ }^{13} \mathrm{C}\left\{{ }^{1} \mathrm{H}\right\} \mathrm{NMR}\left(\mathrm{CDCl}_{3}, 125 \mathrm{MHz}\right): \delta$ 143.4, $140.2,137.6,128.5,128.0,127.8,125.2,108.8,73.6,71.3,67.7$, and $43.3 \mathrm{ppm}$; IR (neat) $3409,3085,3062,3027,2923,2853,1951,1712,1602,1496,1454,1364,1274,1161$, 1080, 1030, 751, and $699 \mathrm{~cm}^{-1}$. HRMS-CI $\mathrm{m} / z 249.1121\left[\mathrm{MH}^{+}\right.$; calcd for $\mathrm{C}_{14} \mathrm{H}_{17} \mathrm{O}_{4}$ : 249.1127].

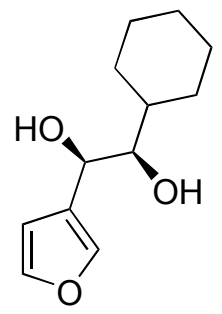

(1R,2R)-1-Cyclohexyl-2-furan-3-yl-ethane-1,2-diol (5c). This compound was prepared by General Procedure D using trans-1-cyclohexyl-2-furan-3yl-ethene (176 mg, $1.0 \mathrm{mmol})$, AD- $\beta$-mix (1.4 g), methanesulfonamide (95 $\mathrm{mg}, 1.0 \mathrm{mmol})$ tert-butyl alcohol $(5 \mathrm{~mL})$ and $\mathrm{H}_{2} \mathrm{O}(5 \mathrm{~mL})$. After the usual workup, the crude product was purified by column chromatography on deactivated silica ( $20 \%$ EtOAc in hexanes) to afford the title compound as a yellow oil in 97\% yield (203 mg, 0.97 mmol). $[\alpha]_{\mathrm{D}}{ }^{20}=-1650.0\left(c=0.002, \mathrm{CHCl}_{3}, 97 \%\right.$ ee $) ;{ }^{1} \mathrm{H} \mathrm{NMR}\left(\mathrm{CDCl}_{3}, 360 \mathrm{MHz}\right) \delta 7.42$ 
(s, 1H), $7.39(\mathrm{~s}, 1 \mathrm{H}), 6.37$ (s, 1H), $4.66(\mathrm{~d}, J=3.6 \mathrm{~Hz}, 1 \mathrm{H}), 4.09$ (q, $J=7.2 \mathrm{~Hz}, 1 \mathrm{H})$, $3.42(\mathrm{t}, J=7.2 \mathrm{~Hz}, 1 \mathrm{H}), 2.01$ (br s, 2H), $1.72(\mathrm{~m}, 5 \mathrm{H}), 1.19(\mathrm{~m}, 5 \mathrm{H}) \mathrm{ppm} ;{ }^{13} \mathrm{C}\left\{{ }^{1} \mathrm{H}\right\} \mathrm{NMR}$ $\left(\mathrm{CDCl}_{3}, 125 \mathrm{MHz}\right): \delta 143.4,140.6,140.0,108.7,78.9,67.3,39.4,30.0,27.3,26.3,26.2$, and $26.0 \mathrm{ppm}$; IR (neat) 3416, 2925, 2852, 2356, 2339, 2093, 1666, 1581, 1366, 1519, $1450,1422,1383,1312,1240,1192,1126,1020,960,893$, and $845 \mathrm{~cm}^{-1}$. HRMS-CI $\mathrm{m} / z$ $193.1232\left[\mathrm{M}-\mathrm{OH}^{+}\right.$; calcd for $\left.\mathrm{C}_{12} \mathrm{H}_{17} \mathrm{O}_{2}: 193.1223\right]$.

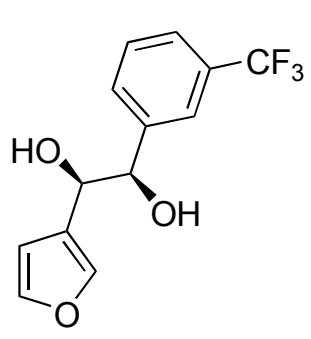

(1R,2R)-1-Furan-3-yl-2-(2-trifloromethyl-phenyl)-ethane-1,2-diol (5d). This compound was prepared by General Procedure D using trans-1-furan-3-yl-2-(2-trifloromethyl-phenyl)-ethene (238 mg, 1.0 mmol), AD $\beta$-mix (1.4 g), methanesulfonamide (95 mg, $1.0 \mathrm{mmol}$ ) tert-butyl alcohol (5 mL) and $\mathrm{H}_{2} \mathrm{O}(5 \mathrm{~mL})$. After the usual workup, the crude product was purified by column chromatography on deactivated silica ( $20 \%$ EtOAc in hexanes) to afford the title compound as a yellow oil in $79 \%$ yield $(242 \mathrm{mg}, 0.79 \mathrm{mmol}) ;[\alpha]_{\mathrm{D}}{ }^{20}=-$ $176.0\left(c=0.025, \mathrm{CHCl}_{3}, 99 \%\right.$ ee); ${ }^{1} \mathrm{H} \mathrm{NMR}\left(\mathrm{CDCl}_{3}, 360 \mathrm{MHz}\right): \delta 7.53(\mathrm{~m}, 2 \mathrm{H}), 7.34(\mathrm{~m}$, 3H), $7.13(\mathrm{~s}, 1 \mathrm{H}), 6.14(\mathrm{~s}, 1 \mathrm{H}), 4.67(\mathrm{~d}, J=9.0 \mathrm{~Hz}, 1 \mathrm{H}), 4.58$ (d, $J=9.0 \mathrm{~Hz}, 1 \mathrm{H}), 3.79$ (br s, 1H), 3.59 (br s, $1 \mathrm{H}){ }^{13} \mathrm{C}\left\{{ }^{1} \mathrm{H}\right\} \mathrm{NMR}\left(\mathrm{CDCl}_{3}, 75 \mathrm{MHz}\right)$ : $\delta$ 163.7, 144.6, 142.3, 141.6, 131.6, 129.7, 125.9, 125.5, 124.9, 109.8, 78.4, 72.5, and $43.7 \mathrm{ppm}$; IR (neat) 3271, 3021, $2935,1707,1616,1449,1416,1330,1162,1126,1074,979,907,806,767,738,702$, and $695 \mathrm{~cm}^{-1}$. HRMS-CI $\mathrm{m} / z 272.0671\left[\mathrm{M}^{+}\right.$; calcd for $\left.\mathrm{C}_{13} \mathrm{H}_{11} \mathrm{~F}_{3} \mathrm{O}_{3}: 272.066\right]$.

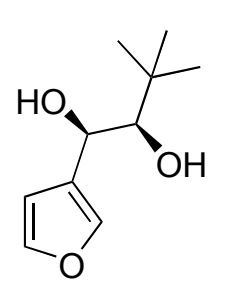

(1R,2R)-1-Furan-3-yl-3, 3-dimethyl-butane-1,2-diol (5e). This compound was prepared by General Procedure D using trans-1-furan-3-yl-3,3-dimethyl- 
butene (150 mg, $1.0 \mathrm{mmol}$ ), AD $\beta$-mix (1.4 g), methanesulfonamide (95 mg, 1.0 mmol) tert-butyl alcohol $(5 \mathrm{~mL})$ and $\mathrm{H}_{2} \mathrm{O}(5 \mathrm{~mL})$. After the usual workup, the crude product was purified by column chromatography on deactivated silica $(20 \%$ EtOAc in hexanes) to afford the title compound as a yellow oil in $89 \%$ yield (164 mg, $0.89 \mathrm{mmol}$ ); $[\alpha]_{\mathrm{D}}{ }^{20}=-1.125\left(c=1.008, \mathrm{CHCl}_{3}, 97 \%\right.$ ee $) ;{ }^{1} \mathrm{H} \mathrm{NMR}\left(\mathrm{CDCl}_{3}, 500 \mathrm{MHz}\right) \delta 7.43(\mathrm{~s}, 1 \mathrm{H})$, $7.40(\mathrm{~s}, 1 \mathrm{H}), 6.42(\mathrm{~s}, 1 \mathrm{H}), 4.79(\mathrm{~s}, 1 \mathrm{H}), 3.35(\mathrm{~s}, 1 \mathrm{H}), 2.76(\mathrm{~s}, 1 \mathrm{H}), 2.72(\mathrm{~s}, 1 \mathrm{H}), 0.99(\mathrm{~s}$, 9H) ppm; ${ }^{13} \mathrm{C}\left\{{ }^{1} \mathrm{H}\right\}$ NMR $\left(\mathrm{CDCl}_{3}, 125 \mathrm{MHz}\right): \delta 143.4,139.7,128.6,108.8,80.9,65.5$, 34.9, and 26.4 ppm; IR (neat) 3417, 3019, 2959, 2870, 2716, 2669, 2471, 2360, 2328, 2095, 1992, 1721, 1665, 1579, 1557, 1503, 1478, 1464, 1392, 1365, 1331, 1159, 1076, 1021, 944, 893, 874, and $778 \mathrm{~cm}^{-1}$. HRMS-CI $\mathrm{m} / z$ 166.0985 [M-OH ${ }^{+}$; calcd for $\mathrm{C}_{10} \mathrm{H}_{14} \mathrm{O}_{2}:$ 166.0994].

\section{$\underline{\text { 3-Formvl furvl alcohol products }}$}

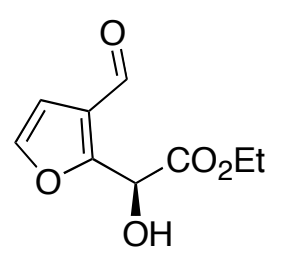

(S)-Ethyl 2-(3-formylfuran-2-yl)-2-hydroxyacetate (6a). This compound was prepared by General Procedure B using 3-furan-3-yl-2,3dihydroxy-propionic acid ethyl ester (48.1 mg, $0.24 \mathrm{mmol})$, THF (1 $\mathrm{mL}), \mathrm{H}_{2} \mathrm{O}(0.25 \mathrm{~mL})$ and NBS $(43 \mathrm{mg}, 0.24 \mathrm{mmol})$. The reaction was quenched by addition of $\mathrm{H}_{2} \mathrm{O}$ and EtOAc. After the organic and aqueous layers were separated, the aqueous layer was extracted with EtOAc $(3 \mathrm{X} 3 \mathrm{~mL})$. The combined organic layers were dried over $\mathrm{MgSO}_{4}$ and filtered. The filtrate was concentrated in vacuo. The residue was purified by column chromatography on silica (10-30\% EtOAc in hexanes) to afford the title compound as a colorless to slightly yellow oil in $63 \%$ yield $(30.0 \mathrm{mg}, 0.151 \mathrm{mmol})$. $[\alpha]_{\mathrm{D}}^{20}=+41.6^{\circ}\left(\mathrm{c}=1.32, \mathrm{CHCl}_{3}, 99 \% \mathrm{ee}\right) ;{ }^{1} \mathrm{H} \mathrm{NMR}\left(\mathrm{CDCl}_{3}, 500 \mathrm{MHz}\right) \delta 10.06(\mathrm{~s}, 1 \mathrm{H})$, 
$7.44(\mathrm{~d}, J=1.8 \mathrm{~Hz}, 1 \mathrm{H}), 6.81(\mathrm{~d}, J=1.8 \mathrm{~Hz}, 1 \mathrm{H}), 5.56(\mathrm{~d}, J=6.7 \mathrm{~Hz}, 1 \mathrm{H}), 4.30(\mathrm{~m}$, $2 \mathrm{H}), 4.11(\mathrm{~d}, J=6.8 \mathrm{~Hz}, 1 \mathrm{H}), 1.27(\mathrm{t}, J=7.1 \mathrm{~Hz}, 3 \mathrm{H}) \mathrm{ppm} ;{ }^{13} \mathrm{C}\left\{{ }^{1} \mathrm{H}\right\} \mathrm{NMR}\left(\mathrm{CDCl}_{3}, 125\right.$ MHz): $\delta 185.5,170.2,157.8,143.6,124.5,109.6,66.8,63.2$, and $14.2 \mathrm{ppm}$; IR (neat) 3445, 3157, 2985, 2930, 1744, 1712, 1683, 1418, 1370, 1296, 1220, 1131, 1095, 1018, and $764 \mathrm{~cm}^{-1}$. HRMS-CI $\mathrm{m} / z 198.0528\left[\mathrm{M}^{+}\right.$; calcd for $\left.\mathrm{C}_{9} \mathrm{H}_{10} \mathrm{O}_{5}: 199.0528\right]$.

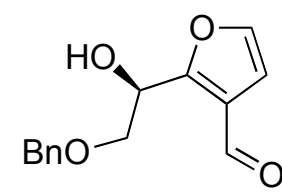

(R)-2-(2-(Benzyloxy)-1-hydroxyethyl)furan-3-carbaldehyde (6b).

This compound was prepared by General Procedure B using 3benzyloxy-1-furan-3-yl-propane-1,2-diol (248 mg, $1.0 \mathrm{mmol}$ ), NBS (178 mg, $1.0 \mathrm{mmol}$ ), THF $(4.0 \mathrm{~mL})$ and $\mathrm{H}_{2} \mathrm{O}(1.0 \mathrm{~mL})$. After the usual workup, the crude product was purified by column chromatography on deactivated silica (10\% EtOAc in hexanes) to afford the title compound as a yellow oil in 55\% yield $(208 \mathrm{mg}, 0.55 \mathrm{mmol})$. $[\alpha]_{\mathrm{D}}{ }^{20}=-173.3(c=$ 0.015, $\mathrm{CHCl}_{3}, 99 \%$ ee). ${ }^{1} \mathrm{H} \mathrm{NMR}\left(\mathrm{CDCl}_{3}, 500 \mathrm{MHz}\right) \delta 10.1(\mathrm{~s}, 1 \mathrm{H}), 7.37(\mathrm{~m}, 6 \mathrm{H}), 6.83$ $(\mathrm{s}, 1 \mathrm{H}), 5.18(\mathrm{q}, J=11.0 \mathrm{~Hz}, 1 \mathrm{H}), 4.65(\mathrm{~s}, 2 \mathrm{H}), 3.84(\mathrm{~d}, J=5.0 \mathrm{~Hz}, 2 \mathrm{H}) \mathrm{ppm} ;{ }^{13} \mathrm{C}\left\{{ }^{1} \mathrm{H}\right\}$ NMR (CDCl $3,125 \mathrm{MHz})$ : $\delta$ 186.6, 161.4, 142.5, 137.4, 128.5, 128.0, 127.8, 124.1, 109.4, 73.5, 72.3, and $67.7 \mathrm{ppm}$; IR (neat) 3640.0, 3156.7, 3078.8, 2926.0, 2853.3, 1827.8, $1782.7,1771.3,1692.3,1451.9,1417.7,1396.7,1369.4,1294.2,1243.5,1190.8,1125.5$, 935.0, 842.0, 821.3, 739.6, 700.0, and $648.4 \mathrm{~cm}^{-1}$. HRMS-CI $\mathrm{m} / z 247.0981\left[\mathrm{MH}^{+}\right.$; calcd for $\left.\mathrm{C}_{14} \mathrm{H}_{15} \mathrm{O}_{4}: 247.0970\right]$.

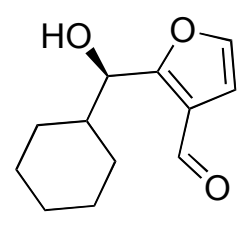

(R)-2-(Cyclohexyl(hydroxy)methyl)furan-3-carbaldehyde (6c). This compound was prepared by General Procedure B using 1-cyclohexyl-2furan-3-yl-ethane-1,2-diol (210 mg, $1.0 \mathrm{mmol})$, NBS (178 mg, 1.0 
mmol), THF $(4.0 \mathrm{~mL})$ and $\mathrm{H}_{2} \mathrm{O}(1.0 \mathrm{~mL})$. After the usual workup, the crude product was purified by column chromatography on deactivated silica $(10 \%$ EtOAc in hexanes) to afford the title compound as a yellow oil in $62 \%$ yield (129 mg, $0.62 \mathrm{mmol})$. $[\alpha]_{\mathrm{D}}{ }^{20}=-0.231\left(c=0.038, \mathrm{CHCl}_{3}, 97 \%\right.$ ee $) ;{ }^{1} \mathrm{H} \mathrm{NMR}\left(\mathrm{CDCl}_{3}, 500 \mathrm{MHz}\right): \delta 9.96(\mathrm{~s}, 1 \mathrm{H})$, $7.35(\mathrm{~s}, 1 \mathrm{H}), 6.73(\mathrm{~s}, 1 \mathrm{H}), 4.66(\mathrm{~d}, J=12.5 \mathrm{~Hz}, 1 \mathrm{H}), 2.00(\mathrm{~m}, 1 \mathrm{H}), 1.78(\mathrm{~m}, 5 \mathrm{H})$, and 1.17 (m, 5H) ppm; ${ }^{13} \mathrm{C}\left\{{ }^{1} \mathrm{H}\right\}$ NMR $\left(\mathrm{CDCl}_{3}, 125 \mathrm{MHz}\right): \delta 187.2,165.3,142.4,123.7,109.7$, 73.2, 44.2, 29.2, 29.1, 26.6, 26.2, and $26.1 \mathrm{ppm}$; IR (neat) 3418.1, 3155.1, 3126.6, 2853.5, 2668.8, 1674.9, 1580.5, 1564.7, 1520.0, 1450.5, 1421.5, 1384.2, 1346.1, 1311.3, $1273.9,1240.7,1193.2,1126.9,1081.8,1032.0,957.8,922.8,893.5,872.2,847.0,804.7$, 763.0, 737.6, 713.6, and $589.7 \mathrm{~cm}^{-1}$. HRMS-CI $\mathrm{m} / z 191.1078$ [M-OH${ }^{+}$; calcd for $\left.\mathrm{C}_{12} \mathrm{H}_{15} \mathrm{O}_{2}: 191.1072\right]$.

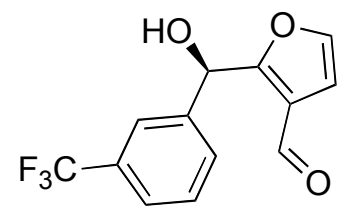

(R)-1-Furan-3-yl-2-(2-triflurormethyl-phenyl)-ethane-1,2-diol

(6d). This compound was prepared by General Procedure B using 1furan-3-yl-2-(2-triflurormethyl-phenyl)-ethane-1,2-diol (272 mg, 1.0 mmol), NBS (178 mg, $1.0 \mathrm{mmol})$, THF $(4.0 \mathrm{~mL})$ and $\mathrm{H}_{2} \mathrm{O}(1.0 \mathrm{~mL})$. After the usual workup, the crude product was purified by column chromatography on deactivated silica (10\% EtOAc in hexanes) to afford the title compound as a yellow oil in $66 \%$ yield (178 $\mathrm{mg}, 0.66 \mathrm{mmol}) ;[\alpha]_{\mathrm{D}}{ }^{20}=-0.561\left(c=0.048, \mathrm{CHCl}_{3}, 88 \%\right.$ ee $) ;{ }^{1} \mathrm{H} \mathrm{NMR}\left(\mathrm{CDCl}_{3}, 500\right.$ MHz) $\delta 9.99(\mathrm{~s}, 1 \mathrm{H}), 7.71(\mathrm{~s}, 1 \mathrm{H}), 7.59(\mathrm{t}, J=10.0 \mathrm{~Hz}, 2 \mathrm{H}), 7.48(\mathrm{t}, J=5.0 \mathrm{~Hz}, 1 \mathrm{H}), 7.37$ (s, 1H), $6.77(\mathrm{~s}, 1 \mathrm{H}), 6.15(\mathrm{~s}, 1 \mathrm{H})$, and $5.18($ br s, $1 \mathrm{H}) \mathrm{ppm} ;{ }^{13} \mathrm{C}\left\{{ }^{1} \mathrm{H}\right\} \mathrm{NMR}\left(\mathrm{CDCl}_{3}, 125\right.$ MHz): $\delta$ 187.4, 162.4, 142.9, 141.1, 130.8, 130.6, 129.6, 129.1, 125.1, 123.0, 110.0, 69.6 and $45.8 \mathrm{ppm}$; IR (neat) 3368, 1673, 1450, 1329, 1165. 1124, 1073, 794, and $701 \mathrm{~cm}^{-1}$. 
HRMS-CI $m / z 270.0496\left[\mathrm{M}^{+}\right.$; calcd for $\left.\mathrm{C}_{13} \mathrm{H}_{9} \mathrm{O}_{3} \mathrm{~F}_{3}: 270.0504\right]$.

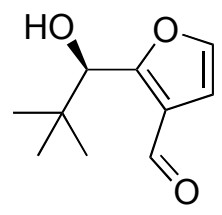

(R)-2-(1-Hydroxy-2,2-dimethyl-propyl)-furan-3-carbaldehyde (6e). This compound was prepared by General Procedure B using 1-furan-3-yl3,3-dimethyl-butane-1,2-diol (184 mg, $1.0 \mathrm{mmol}$ ), NBS (178 mg, 1.0 mmol), THF $(4.0 \mathrm{~mL})$ and $\mathrm{H}_{2} \mathrm{O}(1.0 \mathrm{~mL})$. After the usual workup, the crude product was purified by column chromatography on deactivated silica (10\% EtOAc in hexanes) to afford the title compound as a yellow oil in $59 \%$ yield $(107 \mathrm{mg}, 0.59 \mathrm{mmol}) ;[\alpha]_{\mathrm{D}}{ }^{20}=$ $-5.100\left(c=0.003, \mathrm{CHCl}_{3}, 97 \%\right.$ ee); ${ }^{1} \mathrm{H}$ NMR $\left(\mathrm{CDCl}_{3}, 500 \mathrm{MHz}\right): \delta 9.90(\mathrm{~s}, 1 \mathrm{H}), 7.41(\mathrm{~s}$, $1 \mathrm{H}), 6.81(\mathrm{~s}, 1 \mathrm{H}), 4.66(\mathrm{~s}, 1 \mathrm{H}), 3.88(\mathrm{br} \mathrm{s}, 1 \mathrm{H})$, and $1.00(\mathrm{~s}, 9 \mathrm{H}) \mathrm{ppm} ;{ }^{13} \mathrm{C}\left\{{ }^{1} \mathrm{H}\right\} \mathrm{NMR}$ $\left(\mathrm{CDCl}_{3}, 125 \mathrm{MHz}\right): \delta \quad 187.1,163.6,141.9,123.8,109.9,77.6,37.6$, and $25.7 \mathrm{ppm}$; IR (neat) $3418,2958,2872,1776,1710,1673,1575,1518,1479,1466,1421,1395,1366$, $1293,1240,1181,1129,1068,1016,884,810,778$, and $730 \mathrm{~cm}^{-1}$. HRMS-CI $\mathrm{m} / \mathrm{z}$ $183.1012\left[\mathrm{MH}^{+}\right.$; calcd for $\left.\mathrm{C}_{10} \mathrm{H}_{15} \mathrm{O}_{3}: 183.1021\right]$.

\section{$\underline{\text { Synthesis of Honda's Intermediate for the Synthesis of (-)-Canadensolide }}^{11}$}

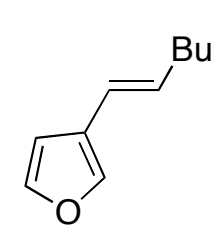

3-Hex-1-enyl-furan (7). Under a nitrogen atmosphere, $\mathrm{PdCl}_{2}(\mathrm{dppf}) \cdot \mathrm{CH}_{2} \mathrm{Cl}_{2}(51 \mathrm{mg}, 0.063 \mathrm{mmol}),(E)$-hexen-1-ylboronic acid (400 mg, $3.13 \mathrm{mmol})$, 3-bromofuran $(277 \mu \mathrm{L}, 3.13 \mathrm{mmol})$, and tertbutylamine ( $986 \mu \mathrm{L}, 9.38 \mathrm{mmol})$ were added to a dry $25 \mathrm{~mL}$ round bottom flask, followed by 2-propanol $(10 \mathrm{~mL})$ and water $(5 \mathrm{~mL})$. A reflux condenser was attached, and the resulting deep red solution was refluxed for $4.5 \mathrm{~h}$ under $\mathrm{N}_{2}$ (oil bath $\approx 90^{\circ} \mathrm{C}$ ). After cooling to rt, water $(10 \mathrm{~mL})$ was added. Following extraction with $\mathrm{Et}_{2} \mathrm{O}(3 \mathrm{X} 10 \mathrm{~mL})$, the 
combined organic layers were washed with $\mathrm{HCl}_{(\mathrm{aq})}(2 \mathrm{~mL}, 2 \mathrm{M})$, brine, dried over $\mathrm{MgSO}_{4}$, filtered, and concentrated under reduced pressure. Column chromatography on silica gel (0-2.5\% EtOAc in hexanes) afforded the title compound in 80\% (1.90 g, 11.4 mmol) as a clear liquid. ${ }^{1} \mathrm{H}$ NMR $\left(\mathrm{CDCl}_{3}, 500 \mathrm{MHz}\right): \delta 7.36(\mathrm{~s}, 2 \mathrm{H}) 6.52(\mathrm{~s}, 1 \mathrm{H}), 6.24$ (d, $J=15.8 \mathrm{~Hz}, 1 \mathrm{H}), 5.96(\mathrm{dt}, J=15.8,6.9 \mathrm{~Hz}, 1 \mathrm{H}) 2.17(\mathrm{dt}, J=6.9,6.6 \mathrm{~Hz}, 2 \mathrm{H}) 1.40$ (m, 4H), $0.94(\mathrm{t}, J=7.0 \mathrm{~Hz}, 3 \mathrm{H}) \mathrm{ppm} ;{ }^{13} \mathrm{C}\left\{{ }^{1} \mathrm{H}\right\} \mathrm{NMR}\left(\mathrm{CDCl}_{3}, 125 \mathrm{MHz}\right): \delta 143.5,139.5$, 131.1, 124.8, 119.4, 107.8, 32.8, 31.7, 22.5, and $14.5 \mathrm{ppm}$; IR (neat) 2958, 2927, 2873, $2859,1508,1466,1379,1162,1072,1025,962,872$, and $771 \mathrm{~cm}^{-1}$. HRMS-CI $\mathrm{m} / \mathrm{z}$ $150.1038\left[\mathrm{M}^{+}\right.$; calcd for $\left.\mathrm{C}_{9} \mathrm{H}_{10} \mathrm{O}_{3}: 150.1045\right]$.

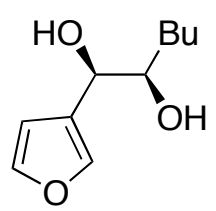

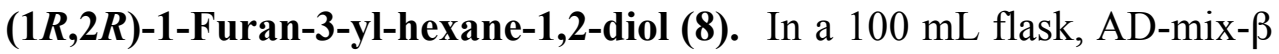
(8.47 g) was dissolved in tert-butanol $(30 \mathrm{~mL})$ and $\mathrm{DI} \mathrm{H}_{2} \mathrm{O}(30 \mathrm{~mL})$ at $\mathrm{rt}$ followed by addition of methanesulfonamide $(575 \mathrm{mg}, 6.05 \mathrm{mmol})$. After dissolution, the orange solution was cooled to $0{ }^{\circ} \mathrm{C}$ and 3-hex-1-enyl-furan (908 $\mathrm{mg}, 6.05$ mmol) was added. After $16 \mathrm{~h}$ of stirring at $0{ }^{\circ} \mathrm{C}$, sodium sulfite $(9.075 \mathrm{~g})$ was added, and the reaction mixture was warmed to ambient temperature and stirred for $1 \mathrm{~h}$. The reaction mixture was extracted with $\mathrm{CH}_{2} \mathrm{Cl}_{2}(3 \mathrm{X} 20 \mathrm{~mL})$, and the combined organic layers were washed with $\mathrm{KOH}(2 \mathrm{~N})$, dried over $\mathrm{MgSO}_{4}$, filtered, and the filtrate was concentrated. Column chromatography on silica gel (10-33\% EtOAc in hexanes) afforded the diol product in $86 \%$ yield $(961 \mathrm{mg}, 5.22 \mathrm{mmol})$ as a clear viscous liquid. $[\alpha]_{\mathrm{D}}{ }^{20}=-3.98^{\circ}\left(\mathrm{c}=2.79, \mathrm{CHCl}_{3},>98 \%\right.$ ee $) ;{ }^{1} \mathrm{H} \mathrm{NMR}\left(\mathrm{CDCl}_{3}, 500 \mathrm{MHz}\right) \delta 7.46(\mathrm{~s}, 1 \mathrm{H})$, $7.43(\mathrm{~s}, 1 \mathrm{H}), 6.42(\mathrm{~s}, 1 \mathrm{H}), 4.46(\mathrm{dd}, J=3.2,5.9 \mathrm{~Hz}, 1 \mathrm{H}), 3.70(\mathrm{~d}, J=6.2 \mathrm{~Hz}, 1 \mathrm{H}), 2.50(\mathrm{~s}$, 1H), $2.33(\mathrm{~s}, 1 \mathrm{H}), 1.39(\mathrm{~m}, 6 \mathrm{H}), 0.90(\mathrm{t}, J=7.1 \mathrm{~Hz}, 3 \mathrm{H}) \mathrm{ppm} ;{ }^{13} \mathrm{C}\left\{{ }^{1} \mathrm{H}\right\} \mathrm{NMR}\left(\mathrm{CDCl}_{3}, 125\right.$ 
MHz): $\delta 143.7,140.4,126.1,108.9,75.2,70.5,32.8,28.0,22.8$, and $14.2 \mathrm{ppm}$; IR (neat) $3391,2957,2934,2873,1595,1503,1467,1380,1161,1055$, and $1026 \mathrm{~cm}^{-1}$. HRMS-CI $m / z$ 167.1064 [(M-OH) ${ }^{+}$; calcd for $\left.\mathrm{C}_{10} \mathrm{H}_{15} \mathrm{O}_{2}{ }^{-}: 167.1072\right]$.

(R)-2-(1-Hydroxy-pentyl)-furan-3-carbaldehyde (9). General Procedure mmol, in two portions $10 \mathrm{~m}$ apart). The yellow solution was stirred for $5 \mathrm{~h}$, quenched with water $(10 \mathrm{~mL})$, extracted with $\mathrm{Et}_{2} \mathrm{O}(3 \mathrm{X} 10 \mathrm{~mL})$, dried over $\mathrm{MgSO}_{4}$, filtered, and the filtrate was concentrated under reduced pressure. The resulting residue was subject to column chromatography on silica gel (5-20\% EtOAc in hexanes) affording the title compound in $68 \%$ yield $(394 \mathrm{mg}, 2.16 \mathrm{mmol}) .[\alpha]_{\mathrm{D}}^{20}=-31.09^{\circ}\left(\mathrm{c}=2.04, \mathrm{CHCl}_{3},>98 \%\right.$ ee); ${ }^{1} \mathrm{H} \mathrm{NMR}\left(\mathrm{CDCl}_{3}, 500 \mathrm{MHz}\right) \delta 10.01(\mathrm{~s}, 1 \mathrm{H}), 7.37(\mathrm{~d}, J=2.0 \mathrm{~Hz}, 1 \mathrm{H}), 6.78(\mathrm{~d}, J=$ $2.0 \mathrm{~Hz}, 1 \mathrm{H}), 4.96(\mathrm{t}, J=6.9 \mathrm{~Hz}, 1 \mathrm{H}), 3.65(\mathrm{~s}, 1 \mathrm{H}), 1.89(\mathrm{~m}, 2 \mathrm{H}), 1.35(\mathrm{~m}, 4 \mathrm{H}), 0.92(\mathrm{t}, J$ $=7.1 \mathrm{~Hz}, 3 \mathrm{H}) \mathrm{ppm} ;{ }^{13} \mathrm{C}\left\{{ }^{1} \mathrm{H}\right\} \mathrm{NMR}\left(\mathrm{CDCl}_{3}, 125 \mathrm{MHz}\right) \delta 187.1,165.4,142.1,122.7$, 110.0, 68.4, 36.1, 27.6, 22.6, and $14.1 \mathrm{ppm}$; IR (film) 3418, 2958, 2863, 1682, 1668, 1427, 1132, and $1048 \mathrm{~cm}^{-1}$. HRMS-CI $\mathrm{m} / z 182.0943\left[\mathrm{M}^{+}\right.$; calcd for $\mathrm{C}_{10} \mathrm{H}_{14} \mathrm{O}_{3}$ : 182.0943].

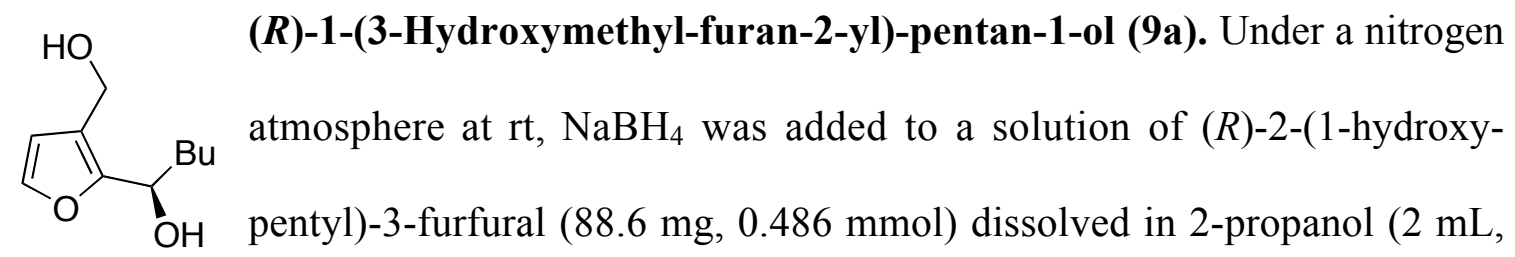
HPLC grade). After stirring for $15 \mathrm{~m}$, the reaction was quenched with $\mathrm{H}_{2} \mathrm{O}(3 \mathrm{~mL})$ and 
$\mathrm{Et}_{2} \mathrm{O}$ was added $(5 \mathrm{~mL})$. The reaction was extracted with $\mathrm{Et}_{2} \mathrm{O}(3 \mathrm{X} 10 \mathrm{~mL})$, dried over $\mathrm{MgSO}_{4}$, filtered, and concentrated under reduced pressure. This material was taken on to the next reaction without purification.

For purposes of characterization, the crude material was run though a plug of silica gel (50\% EtOAc in hexanes) to afford the diol in $95 \%$ yield. $[\alpha]_{\mathrm{D}}{ }^{20}=24.03^{\circ}(\mathrm{c}=$ 3.77, $\left.\mathrm{CHCl}_{3}\right) ;{ }^{1} \mathrm{H} \mathrm{NMR}\left(\mathrm{CDCl}_{3}, 500 \mathrm{MHz}\right) \delta 7.31(\mathrm{~d}, J=1.8 \mathrm{~Hz}, 1 \mathrm{H}), 6.36(\mathrm{~d}, J=1.7 \mathrm{~Hz}$, 1H), $4.78(\mathrm{t}, J=7.0 \mathrm{~Hz}, 1 \mathrm{H}), 4.57$ (s, 2H), 2.67(s, 1H), $2.38(\mathrm{~s}, 1 \mathrm{H}), 1.90(\mathrm{~m}, 2 \mathrm{H}), 1.39-$ $1.25(\mathrm{~m}, 4 \mathrm{H}), 0.92(\mathrm{t}, J=7.1 \mathrm{~Hz}, 3 \mathrm{H}) ;{ }^{13} \mathrm{C}\left\{{ }^{1} \mathrm{H}\right\} \mathrm{NMR}\left(\mathrm{CDCl}_{3}, 125 \mathrm{MHz}\right) \delta$ 153.4, 141.2, 120.8, 111.5, 67.3, 56.7, 35.7, 27.9, 22.7, and $14.2 \mathrm{ppm}$; IR (film) 3335, 2967, 2933, 2873, 2862, 1512, 1467, 1145, 1047, 999, 890, and $730 \mathrm{~cm}^{-1}$. HRMS-ESI $\mathrm{m} / \mathrm{z} 207.0994$ [(M+Na) ${ }^{+}$; calcd for $\mathrm{C}_{10} \mathrm{H}_{16} \mathrm{O}_{3} \mathrm{Na}$ : 207.0997]. The ${ }^{1} \mathrm{H}$ NMR spectrum and the IR for this compound match with previously reported literature data. ${ }^{11}$ The ${ }^{13} \mathrm{C}$ NMR was not previously reported.

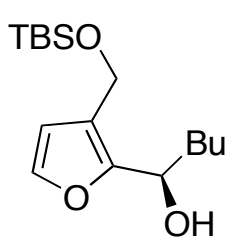

(R)-1-[3-(tert-Butyl-dimethyl-silanyloxymethyl)-furan-2-yl]-pentan-1ol (10). The crude diol (R)-1-(3-Hydroxymethyl-furan-2-yl)-pentan-1-ol (0.486 mmol), TBSCl (73.2 mg, $0.486 \mathrm{mmol})$, and imidazole (66.2 mg, $0.972 \mathrm{mmol})$ were dissolved in $\mathrm{CH}_{2} \mathrm{Cl}_{2}(2 \mathrm{~mL})$ and stirred for $8 \mathrm{~h}$ at $\mathrm{rt}$ under a nitrogen atmosphere. The reaction mixture was quenched with aqueous sat. $\mathrm{NH}_{4} \mathrm{Cl}$ and was extracted with $\mathrm{Et}_{2} \mathrm{O}\left(15 \mathrm{~mL}\right.$ x 3). The combined organic layers were dried over $\mathrm{MgSO}_{4}$, filtered, and the filtrate was concentrated under reduced pressure. Column chromatography on silica gel (5-10\% EtOAc in hexanes) afforded title compound in $78 \%$ yield $(113.1 \mathrm{mg}, 0.379 \mathrm{mmol}) .[\alpha]_{\mathrm{D}}^{20}=9.65^{\circ}\left(\mathrm{c}=2.07, \mathrm{CHCl}_{3}\right) ;{ }^{1} \mathrm{H} \mathrm{NMR}\left(\mathrm{CDCl}_{3}, 500\right.$ 
MHz) $\delta 7.28(\mathrm{~d}, J=1.6 \mathrm{~Hz}, 1 \mathrm{H}), 6.29(\mathrm{~d}, J=1.7 \mathrm{~Hz}, 1 \mathrm{H}), 4.76\left(\mathrm{dd}, J_{1}=6.7,4.8\right.$ Hz, 1H), 4.64 (s, 2H), 2.85 (d, $J=4.7 \mathrm{~Hz}, 1 \mathrm{H}), 1.88(\mathrm{~m}, 2 \mathrm{H}), 1.44-1.26(\mathrm{~m}, 4 \mathrm{H}), 0.93$ (m, 12H), 0.13 (s, 6H) ppm; ${ }^{13} \mathrm{C}\left\{{ }^{1} \mathrm{H}\right\} \mathrm{NMR}\left(\mathrm{CDCl}_{3}, 125 \mathrm{MHz}\right) \delta$ 153.3, 140.7, 120.6 111.2, 67.4, 57.8, 35.7, 27.9, 26.1, 22.8, 18,5, 14.2, -5.08, -5.10 ppm; IR (film) 3412, 2956, 2931, 2856, 1471, 1256, 1148, 1087, 1057, 838, and $777 \mathrm{~cm}^{-1}$. HRMS-ESI $m / z 321.1876$ $\left[(\mathrm{M}+\mathrm{Na})^{+}\right.$; calcd for $\left.\mathrm{C}_{16} \mathrm{H}_{30} \mathrm{O}_{3} \mathrm{SiNa}: 321.1862\right]$. The ${ }^{1} \mathrm{H}$ NMR spectrum, IR, and optical rotation for this compound match those Honda and coworkers. ${ }^{11}$ The ${ }^{13} \mathrm{C}$ NMR was not previously reported.

\section{$\underline{\text { 3-Furyl Tosylamides }}$}

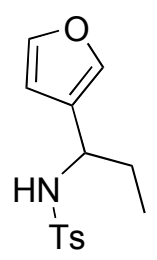

$N$-(1-furan-3-yl-propyl)-4-methyl-benzene-sulfonamide (12a). Th $\mathrm{h}$ i s compound was prepared according to General Procedure A under a nitrogen atmosphere using 3-furyl $N$-tosyl aldimine (249 mg, $1.0 \mathrm{mmol})$, THF (1 mL), and ethyl magnesium bromide $(0.5 \mathrm{~mL}, 3.0 \mathrm{M}$ solution in ether). After quenching with saturated aqueous $\mathrm{NH}_{4} \mathrm{Cl}$, the organic and aqueous layers were separated, and the aqueous layer was extracted with $\mathrm{Et}_{2} \mathrm{O}(3 \times 10 \mathrm{~mL})$. The combined organic layers were dried over $\mathrm{MgSO}_{4}$ and filtered. The filtrate was concentrated in vacuo, and the residue was chromatographed on silica (10\% EtOAc in hexanes) to afford the title compound as a colorless oil in 94\% yield (262 mg, $0.94 \mathrm{mmol}) .{ }^{1} \mathrm{H}$ NMR $\left(\mathrm{CDCl}_{3}, 500 \mathrm{MH}\right) \delta 7.80$ (d, $J$ $=8.1 \mathrm{~Hz}, 2 \mathrm{H}), 7.26(\mathrm{~m}, 3 \mathrm{H}), 7.10(\mathrm{~s}, 1 \mathrm{H}), 6.10(\mathrm{~s}, 1 \mathrm{H}), 4.30(\mathrm{~m}, 1 \mathrm{H}), 4.23(\mathrm{q}, J=7.0 \mathrm{~Hz}$, 1H), $2.44(\mathrm{~s}, 3 \mathrm{H}), 1.75(\mathrm{~m}, 2 \mathrm{H})$, and $0.85(\mathrm{t}, J=7.4 \mathrm{~Hz}, 3 \mathrm{H}) \mathrm{ppm} ;{ }^{13} \mathrm{C}\left\{{ }^{1} \mathrm{H}\right\} \mathrm{NMR}\left(\mathrm{CDCl}_{3}\right.$, $125 \mathrm{MHz}): \delta 143.3,143.2,139.5,137.9,129.4,127.0,125.8,108.3,51.5,29.3,21.5$, and 10.1 ppm; IR (neat) 3356, 3260, 2923, 2302, 1599, 1527, 1450, 1385, 1302, 1157, 1088, 
1063, 1018, 904, 870, 812, and $749 \mathrm{~cm}^{-1}$. HRMS-CI $m / z$ 280.1007 [MH'; calcd for $\left.\mathrm{C}_{14} \mathrm{H}_{18} \mathrm{NO}_{3} \mathrm{~S}: 280.1007\right]$.

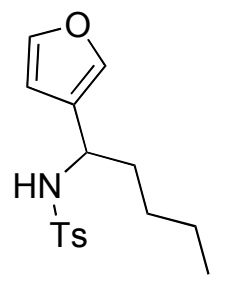

N-(1-furan-3-yl-pentyl)-4-methyl-benzene-sulfonamide (12b). This compound was prepared by General Procedure A using 3-furyl $N$-tosyl aldimine (249 mg, $1.0 \mathrm{mmol})$, THF (1.0 mL), and $n$-butyl lithium $(0.8 \mathrm{~mL}$,

$2.5 \mathrm{M}$ in hexanes, $2.0 \mathrm{mmol})$. After the usual workup, the crude product was purified by column chromatography on deactivated silica (0-10\% EtOAc in hexanes) to afford the title compound as a yellow oil in $90 \%$ yield $(263 \mathrm{mg}, 0.90 \mathrm{mmol}) .{ }^{1} \mathrm{H} \mathrm{NMR}\left(\mathrm{CDCl}_{3}, 500\right.$ MHz) $\delta 7.68(\mathrm{~d}, J=7.2 \mathrm{~Hz}, 2 \mathrm{H}), 7.29(\mathrm{~m}, 3 \mathrm{H}), 7.08(\mathrm{~s}, 1 \mathrm{H}), 6.10(\mathrm{~d}, J=0.5 \mathrm{~Hz}, 1 \mathrm{H})$, $5.08(\mathrm{~d}, J=7.7 \mathrm{~Hz}, 1 \mathrm{H}), 4.28(\mathrm{q}, J=7.2 \mathrm{~Hz}, 1 \mathrm{H}), 2.43(\mathrm{~s}, 3 \mathrm{H}), 1.68(\mathrm{~m}, 2 \mathrm{H}), 1.29(\mathrm{~m}$, 4H), $0.85(\mathrm{t}, J=7.2 \mathrm{~Hz} 3 \mathrm{H}) \mathrm{ppm} ;{ }^{13} \mathrm{C}\left\{{ }^{1} \mathrm{H}\right\} \mathrm{NMR}\left(\mathrm{CDCl}_{3}, 125 \mathrm{MHz}\right): \delta 143.3,143.1$, $139.9,138.8,129.4,127.1,125.5,108.4,50.0,36.0,27.7,22.2,21.5$, and $13.8 \mathrm{ppm}$; IR (neat) $3254,3137,2950,2928,2868,1599,1498,1450,1428,1376,1306,1156,1093$, $1070,1024,873,810,758,684,665,603,581$, and $555 \mathrm{~cm}^{-1}$. HRMS-CI $\mathrm{m} / z 330.1131$ $\left[\mathrm{M}+\mathrm{Na}^{+}\right.$; calcd for $\left.\mathrm{C}_{16} \mathrm{H}_{21} \mathrm{NO}_{3} \mathrm{NaS}: 330.1131\right]$.

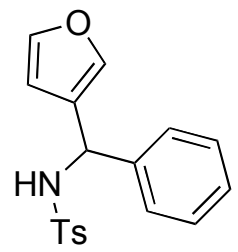

\section{$N$-(1-furan-3-yl-phenyl-methyl-)-4-methyl-benzene-sulfonamide}

(12c). This compound was prepared by General Procedure A using 3furyl tosyl aldimine (249 $\mathrm{mg}, 1.0 \mathrm{mmol}), \operatorname{THF}(1.0 \mathrm{~mL})$ and phenyl lithium (1.0 mL, 2.0 M in hexanes, $2.0 \mathrm{mmol})$. After the usual workup, the crude product was purified by column chromatography on deactivated silica (0-10\% EtOAc in hexanes) to afford the title compound as a yellow oil in 90\% yield (294 mg, $0.90 \mathrm{mmol}) .{ }^{1} \mathrm{H}$ NMR 
$\left(\mathrm{CDCl}_{3}, 500 \mathrm{MHz}\right) \delta 7.61(\mathrm{~d}, J=7.5 \mathrm{~Hz}, 2 \mathrm{H}), 7.30(\mathrm{~d}, J=7.5 \mathrm{~Hz}, 2 \mathrm{H}), 7.24(\mathrm{~m}$, 3H), $7.17(\mathrm{~m}, 3 \mathrm{H}), 7.04(\mathrm{~s}, 1 \mathrm{H}), 6.15(\mathrm{~s}, 1 \mathrm{H}), 5.51(\mathrm{~d}, J=7.7 \mathrm{~Hz}, 1 \mathrm{H}), 2.41(\mathrm{~s}, 3 \mathrm{H}) \mathrm{ppm}$; ${ }^{13} \mathrm{C}\left\{{ }^{1} \mathrm{H}\right\}$ NMR $\left(\mathrm{CDCl}_{3}, 125 \mathrm{MHz}\right): \delta 143.6,143.2,129.4,128.5,127.7,127.1,126.9$, 109.5, 54.1, and $21.5 \mathrm{ppm}$; IR (neat) 3266, 3154, 1597, 1496, 1437, 1324, 1157, 1021, 933, 847, and $811 \mathrm{~cm}^{-1}$. HRMS-CI $\mathrm{m} / z 328.0992 \quad\left[\mathrm{MH}^{+}\right.$; calcd for $\mathrm{C}_{18} \mathrm{H}_{18} \mathrm{NO}_{3} \mathrm{~S}$ : 328.1007].

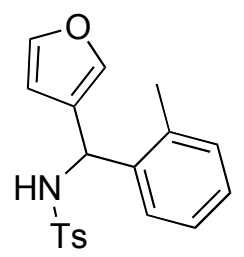

\section{$N$-(1-furan-3-yl-p-tolyl-methyl)-4-methyl-benzene-sulfonamide (12d).}

This compound was prepared by General Procedure A using $o$ bromotoluene (294 mg, $2.0 \mathrm{mmol})$, magnesium turnings (48.6 mg, 2.0 mmol), 3-furyl tosyl imine (249 $\mathrm{mg}, 1.0 \mathrm{mmol})$, and THF (4 mL) were added to the reaction vessel. After the usual workup, the crude product was purified by column chromatography on deactivated silica (10\% EtOAc in hexanes) to afford the title compound as a colorless oil in $91 \%$ yield $(310 \mathrm{mg}, 0.91 \mathrm{mmol}) .{ }^{1} \mathrm{H}$ NMR $\left(\mathrm{CDCl}_{3}, 500\right.$ MHz) $\delta 7.59(\mathrm{~d}, J=10.0 \mathrm{~Hz}, 2 \mathrm{H}), 7.29(\mathrm{~s}, 1 \mathrm{H}), 7.11(\mathrm{~m}, 6 \mathrm{H}), 6.93(\mathrm{~s}, 1 \mathrm{H}), 6.15(\mathrm{~s}, 1 \mathrm{H})$, $5.73(\mathrm{~d}, J=10 \mathrm{~Hz}, 1 \mathrm{H}) 5.34(\mathrm{~s}, 1 \mathrm{H}), 2.39(\mathrm{~s}, 3 \mathrm{H}), 2.24(\mathrm{~s}, 3 \mathrm{H}) \mathrm{ppm} ;{ }^{13} \mathrm{C}\left\{{ }^{1} \mathrm{H}\right\} \mathrm{NMR}$ $\left(\mathrm{CDCl}_{3}, 125 \mathrm{MHz}\right): \delta 143.5,143.2,140.7,137.8,137.5,130.5,129.3,129.1,127.6$, 127.0, 126.6, 126.2, 126.0, 109.7, 50.8, 21.4, and $19.0 \mathrm{ppm}$; IR (neat) 3274, 2957, 2359, $2341,2108,1920,1701,1636,1598,1494,1433,1328,1223,1161,1093,1043,921$, and $874 \mathrm{~cm}^{-1}$. HRMS-CI m/z $342.1153\left[\mathrm{M}^{+}\right.$; calcd for $\left.\mathrm{C}_{19} \mathrm{H}_{19} \mathrm{NO}_{3} \mathrm{~S}: 342.1164\right]$.

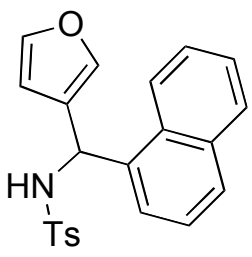

N-(1-furan-3-yl-napthalen-1-yl-methyl)-4-methyl-benzenesulfonamide (12e). This compound was prepared by General 
Procedure A using 1-bromo naphthalene (414 mg, $2.0 \mathrm{mmol})$, magnesium turnings (48.6 mg, $2.0 \mathrm{mmol})$, THF (4 mL) and 3-furyl tosyl aldimine (249 $\mathrm{mg}, 1.0 \mathrm{mmol})$ was added to the reaction vessel. After the usual workup, the residue was chromatographed on silica (10\% EtOAc in hexanes) to afford the title compound as a colorless oil in $92 \%$ yield (347 mg, $0.92 \mathrm{mmol}) .{ }^{1} \mathrm{H}$ NMR $\left(\mathrm{CDCl}_{3}, 360 \mathrm{MHz}\right) \delta 7.91(\mathrm{~m}, 1 \mathrm{H}), 7.82(\mathrm{~m}, 1 \mathrm{H})$, $7.73(\mathrm{~m}, 1 \mathrm{H}), 7.50(\mathrm{~m}, 4 \mathrm{H}), 7.29(\mathrm{~m}, 3 \mathrm{H}), 7.03(\mathrm{~m}, 3 \mathrm{H}), 6.24(\mathrm{~s}, 1 \mathrm{H}), 6.16(\mathrm{~s}, 1 \mathrm{H}), 5.39$ (s, 1H), 2.34 (s, 3H) ppm; ${ }^{13} \mathrm{C}\left\{{ }^{1} \mathrm{H}\right\} \mathrm{NMR}\left(\mathrm{CDCl}_{3}, 75 \mathrm{MHz}\right): \delta$ 143.9, 143.6, 141.3, 137.7, $135.4,134.3,130.7,129.6,129.2,129.1,127.4,126.9,126.7,126.2,125.8,125.5,123.6$, 110.2, 51.9, and $21.8 \mathrm{ppm}$; IR (neat) 3464, 2095, 1703, 1643, 1598, 1510, 1434, 1328, $1224,1158,1091,1022,927$, and $874 \mathrm{~cm}^{-1}$. HRMS-CI $\mathrm{m} / z 377.1103\left[\mathrm{M}^{+}\right.$; calcd for $\left.\mathrm{C}_{22} \mathrm{H}_{19} \mathrm{NO}_{3} \mathrm{~S}: 377.1086\right]$

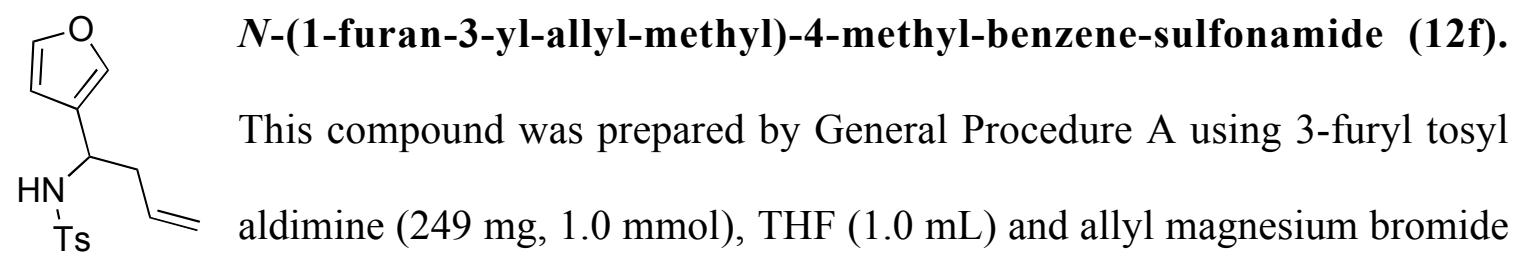
(1.0 mL, 2.0 M in hexanes, $2.0 \mathrm{mmol})$. After the usual workup, the crude product was purified by column chromatography on deactivated silica (0-10\% EtOAc in hexanes) to afford the title compound as a yellow oil in $91 \%$ yield $(265 \mathrm{mg}, 0.91 \mathrm{mmol}) .{ }^{1} \mathrm{H}$ NMR $\left(\mathrm{CDCl}_{3}, 500 \mathrm{MHz}\right) \delta 7.70(\mathrm{~d}, J=8.2 \mathrm{~Hz}, 2 \mathrm{H}), 7.26(\mathrm{~m} \mathrm{3H}), 7.16(\mathrm{~s}, 1 \mathrm{H}), 6.15(\mathrm{~s}, 1 \mathrm{H})$, $5.60(\mathrm{~m}, 1 \mathrm{H}), 5.07(\mathrm{~m}, 2 \mathrm{H}), 4.42(\mathrm{q}, J=7.0 \mathrm{~Hz}, 1 \mathrm{H}), 2.44(\mathrm{~m}, 5 \mathrm{H}) \mathrm{ppm} ;{ }^{13} \mathrm{C}\left\{{ }^{1} \mathrm{H}\right\} \mathrm{NMR}$ $\left(\mathrm{CDCl}_{3}, 125 \mathrm{MHz}\right): \delta 143.3,139.6,137.7,132.9,129.5,127.1,125.4,119.2,118.7,49.4$, 40.4, and $21.5 \mathrm{ppm}$; IR (neat) 2347, 2137, 2987, 1597, 1501, 1452, 1433, 1329, 1161, 1093, 1058, 1023, 991, 929, and $874 \mathrm{~cm}^{-1}$. HRMS-CI $\mathrm{m} / z 291.0917 \quad\left[\mathrm{M}^{+}\right.$; calcd for 
$\left.\mathrm{C}_{15} \mathrm{H}_{17} \mathrm{NO}_{3} \mathrm{~S}: 291.0929\right]$.

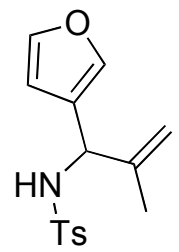

$N$-(1-furan-3-yl-2-isopropenyl-methyl)-4-methyl-benzene-sulfonamide

(12g). This compound was prepared by General Procedure A using 1bromo-2-methyl-2-propene (130 mL, $1.5 \mathrm{mmol})$, THF (4 mL), $n$-butyl lithium ( $0.6 \mathrm{~mL}, 1.5 \mathrm{mmol}, 2.5 \mathrm{M}$ solution in hexanes), 3-furyl tosyl aldimine (249 mg, $1.0 \mathrm{mmol})$. The reaction was stirred at $-78{ }^{\circ} \mathrm{C}$ for $3 \mathrm{~h}$ and quenched with saturated aqueous $\mathrm{NH}_{4} \mathrm{Cl}$. After the usual workup, the residue was chromatographed on silica $(10 \%$ EtOAc in hexanes) to afford the title compound as a colorless oil in 90\% yield (264 mg, $0.90 \mathrm{mmol}) ;{ }^{1} \mathrm{H} \mathrm{NMR}\left(\mathrm{CDCl}_{3}, 500 \mathrm{MHz}\right) \delta 7.73(\mathrm{~d}, J=7.9 \mathrm{~Hz}, 2 \mathrm{H}), 7.29(\mathrm{~m}, 3 \mathrm{H}), 7.16$ (s, 1H), $6.14(\mathrm{~s}, 1 \mathrm{H}), 5.07(\mathrm{~d}, J=7.5 \mathrm{~Hz}, 1 \mathrm{H}), 4.98(\mathrm{~s}, 1 \mathrm{H}), 4.89(\mathrm{~s}, 1 \mathrm{H}), 4.85(\mathrm{~d}, J=7.5$ $\mathrm{Hz}, 1 \mathrm{H}), 2.45$ (s, 3H), and 1.61 (s, 3H) ppm; ${ }^{13} \mathrm{C}\left\{{ }^{1} \mathrm{H}\right\} \mathrm{NMR}\left(\mathrm{CDCl}_{3}, 125 \mathrm{MHz}\right): \delta 143.4$, $143.3,142.9,140.0,137.6,129.4,127.2,124.4,113.6,109.1,55.5,21.5$, and $18.8 \mathrm{ppm}$; IR (neat) $3259,1703,1648,1599,1497,1438,1323,1185,1161,1093,1025$, and 873 $\mathrm{cm}^{-1}$. HRMS-CI $m / z 292.1019$ [MH' $\mathrm{MH}^{+}$calcd for $\left.\mathrm{C}_{15} \mathrm{H}_{18} \mathrm{NO}_{3} \mathrm{~S}: 292.1007\right]$.

\section{2-Subsituted 3-Formyl Pyrroles}

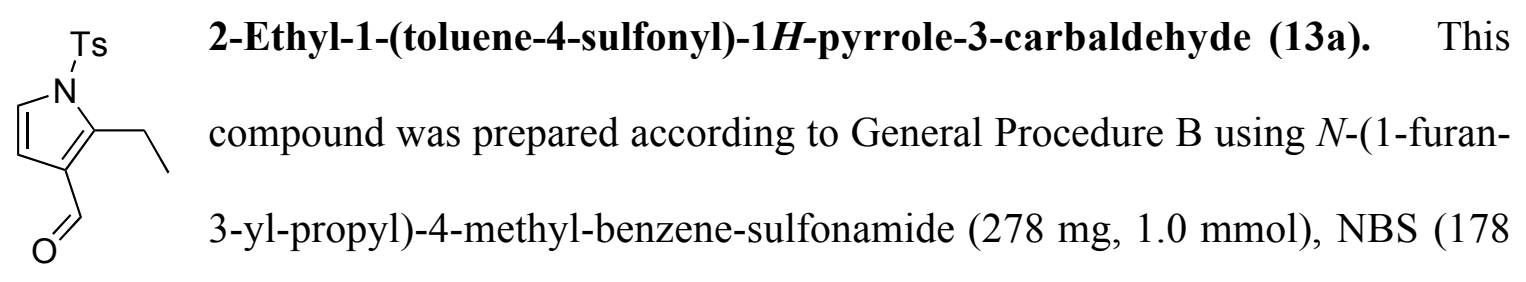

$\mathrm{mg}, 1.0 \mathrm{mmol})$, THF $(4 \mathrm{~mL})$ and $\mathrm{H}_{2} \mathrm{O}(1 \mathrm{~mL})$. After the usual workup, the crude product was purified by column chromatography on deactivated silica (0-10\% EtOAc in hexanes) to afford the title compound as a yellow oil in $67 \%$ yield $(186 \mathrm{mg}, 0.67 \mathrm{mmol}) .{ }^{1} \mathrm{H}$ NMR 
$\left(\mathrm{CDCl}_{3}, 500 \mathrm{MHz}\right) \delta 9.92(\mathrm{~s}, 1 \mathrm{H}), 7.75(\mathrm{~d}, J=5.5 \mathrm{~Hz}, 2 \mathrm{H}), 7.35(\mathrm{~m}, 3 \mathrm{H}), 6.67(\mathrm{~d}, J$ $=4.0 \mathrm{~Hz}, 1 \mathrm{H}), 3.10(\mathrm{q}, J=10.0 \mathrm{~Hz}, 2 \mathrm{H}), 2.47(\mathrm{~s}, 3 \mathrm{H})$, and $1.20(\mathrm{t}, J=10.0 \mathrm{~Hz}, 3 \mathrm{H}) \mathrm{ppm}$; ${ }^{13} \mathrm{C}\left\{{ }^{1} \mathrm{H}\right\}$ NMR $\left(\mathrm{CDCl}_{3}, 125 \mathrm{MHz}\right): \delta 187.1,147.1,146.9,136.8,131.5,128.5,125.4$, 123.7, 110.0, 22.2, 19.3, and 16.5 ppm; IR (neat) 3261, 2976, 2350, 1724, 1596, 1493, $1453,1401,1363,1164,1123,1089,1035,1010,814,703$, and $673 \mathrm{~cm}^{-1}$. HRMS-CI $\mathrm{m} / z$ $277.0760\left[\mathrm{M}^{+}\right.$; calcd for $\left.\mathrm{C}_{14} \mathrm{H}_{15} \mathrm{NO}_{3} \mathrm{~S}: 277.0773\right]$.

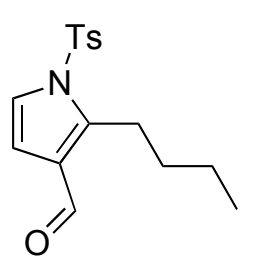

\section{2-Butyl-1-(toluene-4-sulfonyl)-1H-pyrrole-3-carbaldehyde (13b). This} compound was prepared by General Procedure B using N-(1-furan-3-ylpentyl)-4-methyl-benzene-sulfonamide (306 mg, $1.0 \mathrm{mmol})$, NBS (178 $\mathrm{mg}, 1.0 \mathrm{mmol})$, THF $(4 \mathrm{~mL})$ and $\mathrm{H}_{2} \mathrm{O}(1 \mathrm{~mL})$. After the usual workup, the crude product was purified by column chromatography on deactivated silica (0-10\% EtOAc in hexanes) to afford the title compound as a yellow oil in $67 \%$ yield $(204 \mathrm{mg}, 0.67 \mathrm{mmol}) .{ }^{1} \mathrm{H}$ NMR $\left(\mathrm{CDCl}_{3}, 500 \mathrm{MHz}\right) \delta 9.90(\mathrm{~s}, 1 \mathrm{H}), 7.89(\mathrm{~d}, J=8.0 \mathrm{~Hz}, 2 \mathrm{H}), 7.35(\mathrm{~m}, 3 \mathrm{H}), 6.63(\mathrm{~d}, J=3.4$ $\mathrm{Hz}, 1 \mathrm{H}), 2.57(\mathrm{t}, J=7.0 \mathrm{~Hz}, 2 \mathrm{H}), 2.47(\mathrm{~s}, 3 \mathrm{H}), 1.67(\mathrm{~m}, 2 \mathrm{H}), 1.53(\mathrm{~m}, 2 \mathrm{H}), 0.99(\mathrm{t}, J=$ $10.0 \mathrm{~Hz}, 3 \mathrm{H}) \mathrm{ppm} ;{ }^{13} \mathrm{C}\left\{{ }^{1} \mathrm{H}\right\} \mathrm{NMR}\left(\mathrm{CDCl}_{3}, 125 \mathrm{MHz}\right): \delta 185.5,146.1,130.3,130.0$, $128.0,127.0,123.2,108.4,103.2,30.1,22.0,21.7,19.6$, and $13.6 \mathrm{ppm}$; IR (neat) 3434 , $2958,2870,2359,2341,2225,2084,1651,1432,1377,1305,1193,1175,1134,1088$, and $812 \mathrm{~cm}^{-1}$. HRMS-CI $\mathrm{m} / z 330.1140\left[\mathrm{M}+\mathrm{Na}^{+}\right.$; calcd for $\mathrm{C}_{16} \mathrm{H}_{21} \mathrm{NO}_{3} \mathrm{NaS}: 330.1140$ )

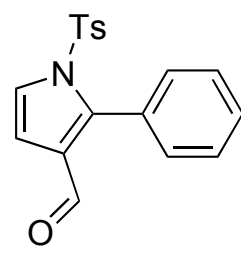

2-Phenyl-1-(toluene-4-sulfonyl)-1H-pyrrole-3-carbaldehyde (13c).

This compound was prepared by General Procedure B using $N$-(1-furan3-yl-phenyl-methyl-)-4-methyl-benzene-sulfonamide (326 mg, 1.0 
mmol), NBS (178 mg, $1.0 \mathrm{mmol})$, THF $(4 \mathrm{~mL})$ and $\mathrm{H}_{2} \mathrm{O}(1 \mathrm{~mL})$. After the usual workup, the crude product was purified by column chromatography on deactivated silica $(0-10 \%$ EtOAc in hexanes) to afford the title compound as a yellow oil in $70 \%$ yield (228 mg, $0.70 \mathrm{mmol}) .{ }^{1} \mathrm{H} \mathrm{NMR}\left(\mathrm{CDCl}_{3}, 300 \mathrm{MHz}\right) \delta 9.35$ (s, 1H), 7.50 (m, 2H), 7.36 (m, 2H), $7.24(\mathrm{~m}, 6 \mathrm{H})$ and $6.77(\mathrm{~s}, 1 \mathrm{H}), 2.49(\mathrm{~s}, 3 \mathrm{H}) \mathrm{ppm} ;{ }^{13} \mathrm{C}\left\{{ }^{1} \mathrm{H}\right\}$ NMR $\left(\mathrm{CDCl}_{3}, 75 \mathrm{MHz}\right)$ : $\delta 188.1,147.0,136.1,133.4,131.0,130.9,129.3,128.9,128.8,128.3,124.8,109.5$, and 21.8 ppm; IR (neat) 3319, 1674, 1595, 1471, 1448, 1424, 1376, 1303, 1190, 1176, 1127 , 1090, 955, and $813 \mathrm{~cm}^{-1}$. HRMS-CI $\mathrm{m} / z 325.0758\left[\mathrm{M}^{+}\right.$; calcd for $\mathrm{C}_{18} \mathrm{H}_{15} \mathrm{NO}_{3} \mathrm{~S}$ : 325.0773].

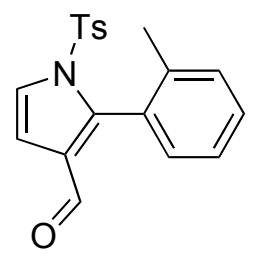

\section{2-Tolyl-1-(toluene-4-sulfonyl)-2-p-tolyl-1H-pyrrole-3-carbaldehyde}

(13d). This compound was prepared by General Procedure B using $N$ (1-furan-3-yl-p-tolyl-methyl)-4-methyl-benzene-sulfonamide (340 mg, $1.0 \mathrm{mmol}$ ), NBS (178 mg, $1.0 \mathrm{mmol})$, THF $(4 \mathrm{~mL})$ and $\mathrm{H}_{2} \mathrm{O}(1 \mathrm{~mL})$. After the usual workup, the crude product was purified by column chromatography on deactivated silica $(0-10 \%$ EtOAc in hexanes) to afford the title compound as a yellow oil in $73 \%$ yield $(247 \mathrm{mg}, 0.73 \mathrm{mmol}$ ); ${ }^{1} \mathrm{H}$ NMR $\left(\mathrm{CDCl}_{3}, 500 \mathrm{MHz}\right): \delta 9.27$ (s, 1H), $7.55(\mathrm{~d}, J=5.0 \mathrm{~Hz}, 1 \mathrm{H}), 7.41(\mathrm{~m}, 1 \mathrm{H}), 7.30$ (m, 2H) $7.23(\mathrm{~m}, 4 \mathrm{H}), 6.94(\mathrm{~d}, J=5.5 \mathrm{~Hz}, 1 \mathrm{H}), 6.80(\mathrm{~d}, J=3.6 \mathrm{~Hz}, 1 \mathrm{H}), 2.44(\mathrm{~s}, 3 \mathrm{H})$, 1.86 (s, 3H) ppm; ${ }^{13} \mathrm{C}\left\{{ }^{1} \mathrm{H}\right\}$ NMR $\left(\mathrm{CDCl}_{3}, 125 \mathrm{MHz}\right): \delta 186.3,145.8,142.0,140.1,134.8$, $132.3,130.2,129.7,128.0,127.8,126.8,124.8,124.8,123.2,108.1,21.7$, and $19.9 \mathrm{ppm}$ IR (neat) 3445, 2958, 1771, 1708, 1676, 1595, 1492, 1423, 1375, 1300, 1191, 1177, $1129,1090,1036,1010,958,814$, and $758 \mathrm{~cm}^{-1}$. HRMS-CI $\mathrm{m} / \mathrm{z} 339.0929$ [M; calcd for $\left.\mathrm{C}_{19} \mathrm{H}_{17} \mathrm{NO}_{3} \mathrm{~S}: 339.0929\right]$. 


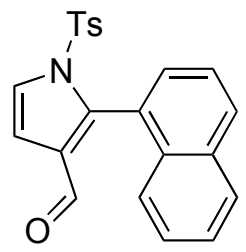

\section{2-Naphthalen-1-yl-1-(toluene-4-sulfonyl)-1 $H$-pyrrole-3-carbaldehyde}

(13e). This compound was prepared by General Procedure B using $N$-(1furan-3-yl-napthalen-1-yl-methyl)-4-methyl-benzene-sulfonamide (376 mg, $1.0 \mathrm{mmol})$, NBS (178 mg, $1.0 \mathrm{mmol})$, THF (4 mL) and $\mathrm{H}_{2} \mathrm{O}(1 \mathrm{~mL})$. After the usual workup, the crude product was purified by column chromatography on deactivated silica $(0-10 \%$ EtOAc in hexanes) to afford the title compound as a yellow oil in $71 \%$ yield (266 mg, $0.71 \mathrm{mmol}) ;{ }^{1} \mathrm{H} \mathrm{NMR}\left(\mathrm{CDCl}_{3}, 500 \mathrm{MHz}\right) \delta 9.09$ (s, 1H), 8.01 (d, $\left.J=10.0 \mathrm{~Hz}, 1 \mathrm{H}\right)$, $7.85(\mathrm{~d}, J=10.0 \mathrm{~Hz}, 1 \mathrm{H}), 7.68(\mathrm{~d}, J=5.0 \mathrm{~Hz}, 1 \mathrm{H}), 7.55(\mathrm{t}, J=10.0 \mathrm{~Hz}, 1 \mathrm{H}), 7.47(\mathrm{~d}, J=$ $10.0 \mathrm{~Hz}, 1 \mathrm{H}), 7.41(\mathrm{t}, J=10.0 \mathrm{~Hz}, 1 \mathrm{H}), 7.14(\mathrm{t}, J=10.0 \mathrm{~Hz}, 1 \mathrm{H}), 7.05(\mathrm{~d}, J=10.0 \mathrm{~Hz}$, 2H), $6.93(\mathrm{~d}, J=10.0 \mathrm{~Hz}, 2 \mathrm{H}), 6.83(\mathrm{~d}, J=10.0 \mathrm{~Hz}, 2 \mathrm{H})$, and $2.23(\mathrm{~s}, 3 \mathrm{H}) \mathrm{ppm} ;{ }^{13} \mathrm{C}\left\{{ }^{1} \mathrm{H}\right\}$ $\mathrm{NMR}\left(\mathrm{CDCl}_{3}, 125 \mathrm{MHz}\right): \delta 186.3,145.5 .140 .2,133.9,133.9,132.8,131.5,130.6,130.5$, $129.3,128.9,128.0,127.7,126.7,125.8,125.1,124.4,123.5,107.9$, and 21.4 ppm; IR (neat) $3301,2088,1702,1633,1598,1511,1494,1432,1330,1229,1163,1092,909$, and $874 \mathrm{~cm}^{-1}$. HRMS-CI $\mathrm{m} / z$ 375.0935[M $\mathrm{M}^{+}$; calcd for $\left.\mathrm{C}_{22} \mathrm{H}_{17} \mathrm{NO}_{3} \mathrm{~S}: 375.0929\right]$.

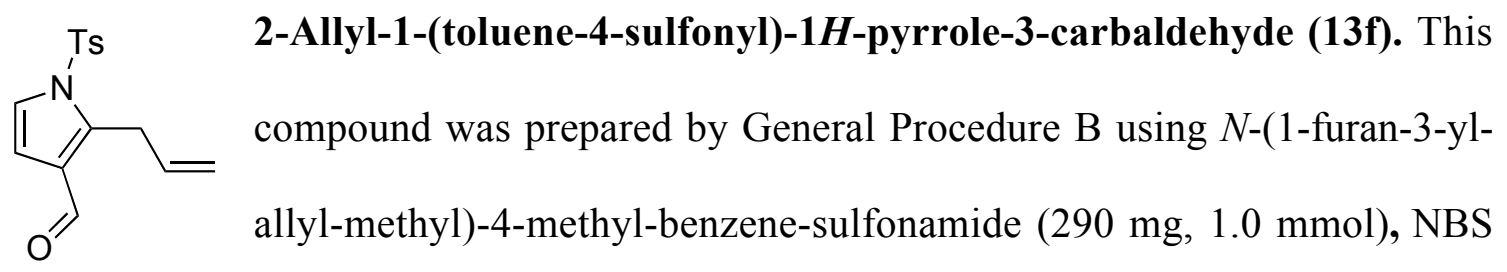
(178 mg, $1.0 \mathrm{mmol})$, THF $(4 \mathrm{~mL})$ and $\mathrm{H}_{2} \mathrm{O}(1 \mathrm{~mL})$. After the usual workup, the crude product was purified by column chromatography on deactivated silica (0-10\% EtOAc in hexanes) to afford the title compound as a yellow oil in $54 \%$ yield ( $289 \mathrm{mg}, 0.54 \mathrm{mmol})$. ${ }^{1} \mathrm{H} \mathrm{NMR}\left(\mathrm{CDCl}_{3}, 360 \mathrm{MHz}\right) \delta 9.90(\mathrm{~s}, 1 \mathrm{H}), 7.74(\mathrm{~d}, J=8.4 \mathrm{~Hz}, 2 \mathrm{H}), 7.35(\mathrm{~m}, 3 \mathrm{H}), 6.69$ 
$(\mathrm{d}, J=3.5 \mathrm{~Hz}, 1 \mathrm{H}), 5.81(\mathrm{~m}, 1 \mathrm{H}), 4.97(\mathrm{~d}, J=11.2 \mathrm{~Hz}, 1 \mathrm{H}), 4.89(\mathrm{~d}, J=18.2 \mathrm{~Hz}$ $1 \mathrm{H}), 3.89(\mathrm{~d}, J=5.8 \mathrm{~Hz}, 2 \mathrm{H})$, and $2.45(\mathrm{~s}, 3 \mathrm{H}) \mathrm{ppm} ;{ }^{13} \mathrm{C}\left\{{ }^{1} \mathrm{H}\right\} \operatorname{NMR}\left(\mathrm{CDCl}_{3}, 125 \mathrm{MHz}\right)$ : $\delta 185.6,145.9,134.1,130.5,130.2,127.3,127.0,123.3,122.9,116.9,109.9,28.8$, and $21.7 \mathrm{ppm}$; IR (neat) 3454, 2955, 1708, 1641, 1427, 1333, 1297, 1160, 1091, and $815 \mathrm{~cm}^{-}$ ${ }^{1}$. HRMS-ESI $m / z$ 314.0815 $\left[\mathrm{MNa}^{+}\right.$; calcd for $\mathrm{C}_{15} \mathrm{H}_{17} \mathrm{NO}_{3} \mathrm{NaS}: 314.0827$ ].

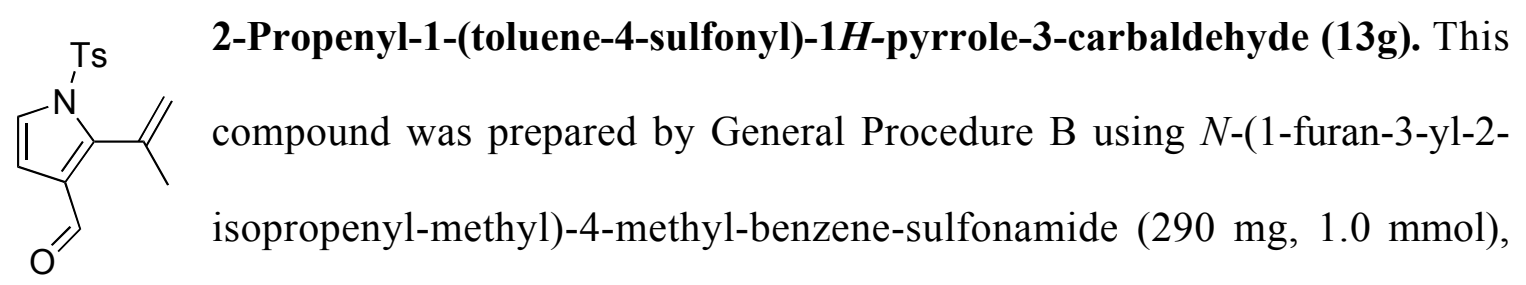
NBS (178 mg, $1.0 \mathrm{mmol})$, THF $(4 \mathrm{~mL})$ and $\mathrm{H}_{2} \mathrm{O}(1 \mathrm{~mL})$. After the usual workup, the crude product was purified by column chromatography on deactivated silica $(0-10 \%$ EtOAc in hexanes) to afford the title compound as a yellow oil in $72 \%$ yield ( $208 \mathrm{mg}$, $0.72 \mathrm{mmol}) ;{ }^{1} \mathrm{H}$ NMR $\left(\mathrm{CDCl}_{3}, 500 \mathrm{MHz}\right) \delta 9.68(\mathrm{~s}, 1 \mathrm{H}), 7.73(\mathrm{~d}, J=8.3 \mathrm{~Hz}, 2 \mathrm{H}), 7.31$ (m, 3H), $6.69(\mathrm{~d}, J=3.4 \mathrm{~Hz}, 1 \mathrm{H}), 5.53(\mathrm{~s}, 1 \mathrm{H}), 4.81(\mathrm{~s}, 1 \mathrm{H}), 2.45(\mathrm{~s}, 3 \mathrm{H}), 2.19(\mathrm{~s}, 3 \mathrm{H})$ ppm; ${ }^{13} \mathrm{C}\left\{{ }^{1} \mathrm{H}\right\}$ NMR $\left(\mathrm{CDCl}_{3}, 125 \mathrm{MHz}\right): \delta$ 186.2, 145.8, 145.2, 135.3, 134.3, 129.9, 127.4, 126.6, 123.3, 123.1, 109.4, 25.3, and 21.7 ppm; IR (neat) 3276, 2923, 2350, 1759, $1709,1650,1597,1556,1537,1494,1445,1330,1225,1160,1092,1022,921,874,814$, and $706 \mathrm{~cm}^{-1}$. HRMS-CI $m / z 289.0769\left[\mathrm{M}^{+}\right.$; calcd for $\mathrm{C}_{15} \mathrm{H}_{15} \mathrm{NO}_{3} \mathrm{~S}: 289.0773$ ].

\section{Conditions for the Determination of Enantiomeric Excess}

The enantiomeric excess values for the following furyl diols and furyl formyl alcohols were determined by chiral HPLC analysis. The conditions for the resolution of the racemates are described below. 


\section{Furyl diols}

3-Furan-3-yl-2,3-dihydroxy-propionic acid ethyl ester (5a). $\mathrm{t}_{1}=17.2 \mathrm{~min}, \mathrm{t}_{2}=20.3$ min (hexanes/2-propanol: 98/2, $1 \mathrm{~mL} / \mathrm{min}, \mathrm{AD}$ column).

3-Benzyloxy-1-furan-3-yl-propane-1,2-diol (5b). $\mathrm{t}_{1}=23.7 \mathrm{~min}, \mathrm{t}_{2}=26.4 \mathrm{~min}$ (hexane $/$ 2-propanol: 90/10, $1.0 \mathrm{~mL} / \mathrm{min}, \mathrm{AD}-\mathrm{H}$ column).

1-Cyclohexyl-2-furan-3-yl-ethane-1,2-diol (5c). $\mathrm{t}_{1}=24.4 \mathrm{~min}, \mathrm{t}_{2}=28.4 \mathrm{~min}$ (hexane $/$ 2-propanol: 99/1, $0.5 \mathrm{~mL} / \mathrm{min}, \mathrm{AD}-\mathrm{H}$ column).

1-Furan-3-yl-2-(2-triflurormethyl-phenyl)-ethane-1,2-diol (5d). $t_{1}=40.1 \mathrm{~min}, \mathrm{t}_{2}=$ $45.7 \mathrm{~min}$ (hexane / 2-propanol: 97/3, $0.75 \mathrm{~mL} / \mathrm{min}$, AD column).

1-Furan-3-yl-3,3-dimethyl-butane-1,2-diol (5e). $\mathrm{t}_{1}=16.6 \mathrm{~min}, \mathrm{t}_{2}=24.7 \mathrm{~min}$ (hexane $/$ 2-propanol: 99/1 $0.5 \mathrm{~mL} / \mathrm{min}, \mathrm{AD}-\mathrm{H}$ column).

1-Furan-3-yl-hexane-1,2-diol (8). $\mathrm{t}_{1}=158 \mathrm{~min}, \mathrm{t}_{2}=169 \mathrm{~min}\left(110^{\circ} \mathrm{C}\right.$, Supelco Beta $\mathrm{DEX}^{\mathrm{TM}} 120$ fused silica column, $30 \mathrm{~m} \times 0.25 \mathrm{~mm} \times 0.25 \mu \mathrm{m}$ film thickness).

\section{Furyl formyl alcohols}

(3-Formyl-furan-2-yl)-hydroxy-acetic acid ethyl ester (6a). $\mathrm{t}_{1}=55.7 \mathrm{~min}, \mathrm{t}_{2}=59.4$ min (hexanes/2-propanol: 90/10, $0.95 \mathrm{~mL} / \mathrm{min}, \mathrm{AD}-\mathrm{H}$ column).

2-(Benzyloxy-hydroxy-methyl)-furan-3-carbaldehyde (6b). $\mathrm{t}_{1}=36.9 \mathrm{~min}, \mathrm{t}_{2}=40.6$ min (hexane / 2-propanol: 97/3, $0.75 \mathrm{~mL} / \mathrm{min}, \mathrm{AD}-\mathrm{H}$ column).

2-(Cyclohexyl-hydroxy-methyl)-furan-3-carbaldehyde (6c). $\mathrm{t}_{1}=28.8 \mathrm{~min}, \mathrm{t}_{2}=38.0$ min (hexane / 2-propanol: 95/5, $0.75 \mathrm{~mL} / \mathrm{min}, \mathrm{AD}-\mathrm{H}$ column).

2-[Hydroxy-(2-trifluoromethyl-phenyl)-methyl]-furan-3-carbaldehyde (6d). $\mathrm{t}_{1}=25.2$ $\min , \mathrm{t}_{2}=42.4 \mathrm{~min}$ (hexane / 2-propanol: 97/3, $0.5 \mathrm{~mL} / \mathrm{min}, \mathrm{AD}$ column).

2-(1-Hydroxy-2, 2-dimethyl-propyl)-furan-3-carbaldehyde (6e). $t_{1}=26.6 \mathrm{~min}, \mathrm{t}_{2}=$ $30.2 \mathrm{~min}$ (hexane / 2-propanol: 95/5, $0.75 \mathrm{~mL} / \mathrm{min}$, AD-H column).

2-(1-Hydroxy-pentyl)-furan-3-carbaldehyde (9). $t_{1}=42.6 \mathrm{~min}, t_{2}=45.9 \mathrm{~min}$ (hexanes/2-propanol: 98/2, $0.75 \mathrm{~mL} / \mathrm{min}, \mathrm{AD}-\mathrm{H}$ column).

\section{References}

(1) Oppolzer, W.; Radinov, R. N. Helv. Chem. Acta. 1992, 75, 170-173.

(2) Langer, F.; Schwink, L.; $\quad$ Devasagayaraj, A.; Chavant, P.-Y.; 
Knochel, P. J. Org. Chem. 1996, 61, 8229-8243.

(3) Jeon, S.-J.; Fisher, E. L.; Carroll, P. J.; Walsh, P. J. J. Am. Chem. Soc. 2006, 128, 9618-9619.

(4) Lu, W.; Chan, T. H. J. Org. Chem. 2001, 66, 3467-3473.

(5) Kitamura, T.; Kiawakami, Y.; Imagawa, T.; Kawanisi, M. Synth. Commun. 1977, 7, 521-528.

(6) Kusababe, M.; Kitano, Y.; Kobayashi, Y.; Sato, F. J. Org. Chem. 1989, 54, 2085-2091.

(7) Pawlicki, M.; Latos-Grazynski, L.; Szterenberg, L. J. Org. Chem. 2002, 67, 5644-5653.

(8) Comins, D. L.; Killpack, M. O. J. Org. Chem. 1987, 52, 104-109.

(9) Quenching at $-78^{\circ} \mathrm{C}$ resulted in $80 \%$ deuterium incorporation at the 2position, where as quenching at room temperature resulted in full deuterium incorporation by crude NMR. The $80 \%$ isotope labeled 2-deutero-3-furaldehyde was carried through the following 2 steps, where $80 \%$ deuterium labeling was also observed for each subsequent product. 222.

(10) Gronowitz, S.; Johnson, I.; Hornfeldt, A.-B. Chem. Scripta 1975, 7, 211 -

(11) Honda, T.; Kobayashi, Y.; Tsubuki, M. Tetrahedron 1993, 49, 1211-1222. 